\title{
Current evidence for COVID-19 therapies: a systematic literature review
}

\author{
Tobias Welte (1) ${ }^{1}$, Lucy J. Ambrose ${ }^{2}$, Gillian C. Sibbring ${ }^{3}$, Shehla Sheikh ${ }^{4}$, \\ Hana Müllerová ${ }^{4}$ and lan Sabir ${ }^{4}$
}

Affiliations: ${ }^{1}$ Dept of Pulmonary and Infectious Diseases, Hannover University School of Medicine, Germany. ${ }^{2}$ Prime Global, Oxford, UK. ${ }^{3}$ Prime Global, Knutsford, UK. ${ }^{4}$ AstraZeneca, Cambridge, UK.

Correspondence: Tobias Welte, Dept of Pulmonary and Infectious Diseases, Hannover University School of Medicine, Welfengarten 1, 30167 Hannover, Germany. E-mail: Welte.Tobiasamh-hannover.de

@ERSpublications

This review shows the need for well-designed trials of treatments for COVID-19 to improve the quality of available evidence and emphasises the importance of tailoring interventions to disease stage and severity for maximum efficacy https://bit.ly/3u72MHa

Cite this article as: Welte T, Ambrose LJ, Sibbring GC, et al. Current evidence for COVID-19 therapies: a systematic literature review. Eur Respir Rev 2021; 30: 200384 [https://doi.org/10.1183/16000617.0384-2020].

ABSTRACT Effective therapeutic interventions for the treatment and prevention of coronavirus disease 2019 (COVID-19) are urgently needed. A systematic review was conducted to identify clinical trials of pharmacological interventions for COVID-19 published between 1 December 2019 and 14 October 2020. Data regarding efficacy of interventions, in terms of mortality, hospitalisation and need for ventilation, were extracted from identified studies and synthesised qualitatively.

In total, 42 clinical trials were included. Interventions assessed included antiviral, mucolytic, antimalarial, anti-inflammatory and immunomodulatory therapies. Some reductions in mortality, hospitalisation and need for ventilation were seen with interferons and remdesivir, particularly when administered early, and with the mucolytic drug, bromhexine. Most studies of lopinavir/ritonavir and hydroxychloroquine did not show significant efficacy over standard care/placebo. Dexamethasone significantly reduced mortality, hospitalisation and need for ventilation versus standard care, particularly in patients with severe disease. Evidence for other classes of interventions was limited. Many trials had a moderate-to-high risk of bias, particularly in terms of blinding; most were short-term and some included low patient numbers.

This review highlights the need for well-designed clinical trials of therapeutic interventions for COVID-19 to increase the quality of available evidence. It also emphasises the importance of tailoring interventions to disease stage and severity for maximum efficacy.

\section{Introduction}

Coronavirus disease 2019 (COVID-19) is an infectious disease caused by a newly discovered coronavirus, severe acute respiratory syndrome coronavirus 2 (SARS-CoV-2) [1]. The global pandemic caused by COVID-19 is ongoing, with a projected death toll of almost 3.5 million by 1 May 2021 [2].

Although COVID-19 presents primarily as a respiratory tract infection, increasing data have shown the potential for systemic involvement, including cardiovascular, neurological and dermatological manifestations, in patients who present with the disease [3]. The pathophysiological course of COVID-19 has been proposed to comprise three distinct phases [4]. In the early infection phase, the SARS-CoV-2

This article has supplementary material available from err.ersjournals.com

Provenance: Submitted article, peer reviewed

Received: 4 Dec 2020 | Accepted: 10 Feb 2021

Copyright (CThe authors 2021. This version is distributed under the terms of the Creative Commons Attribution NonCommercial Licence 4.0. For commercial reproduction rights and permissions contact permissions@ersnet.org 
virus enters epithelial cells in the nasal cavity and multiplies in the upper respiratory tract, with or without pulmonary involvement $[1,4,5]$. The second phase is characterised by localised pulmonary inflammation and the development of viral pneumonia, with or without hypoxia. In a minority of patients, the disease enters a third "host response" phase, which manifests as an extrapulmonary systemic hyperinflammation syndrome, characterised by high levels of pro-inflammatory cytokines and potentially leading to thrombotic complications, viral sepsis and multi-organ failure $[1,4,6]$.

Increasing understanding of the disease pathways involved in COVID-19 has highlighted the importance of selecting and implementing treatments appropriate for the disease stage that patients present with $[4,6]$. Since the start of the outbreak, global efforts to validate effective therapeutic interventions for COVID-19 have resulted in the identification of many potential candidates and the initiation of thousands of clinical trials of therapies with diverse mechanisms of action [7-9].

As of February 2021, several therapies have received regulatory approval on the basis of promising results (figure 1a) [10,11], these include: the antiviral remdesivir; remdesivir in combination with baricitinib (a Janus kinase inhibitor); dexamethasone (a corticosteroid); convalescent plasma; bamlanivimab (a monoclonal antibody therapy); and casirivimab and imdevimab (a cocktail of two monoclonal antibodies) [10-13]. Additionally, several vaccines against SARS-CoV-2 have been approved by the US Food and Drug Administration (FDA) and/or European Medicines Agency [10,11] and other vaccines are in late-phase clinical trials. Despite these developments, treatment options for COVID-19 remain limited. The precise proportion of patients requiring hospitalisation is challenging to determine, given the uncertain prevalence of infection [14]. However, it is estimated that up to $20 \%$ of patients with COVID-19 have an illness severe enough to warrant hospitalisation, which may require intensive care admission and need for respiratory support [15-18]. Consequently, the COVID-19 pandemic has put substantial pressure on healthcare systems worldwide [19-23], and there remains an urgent need for effective agents for the prevention and treatment of COVID-19.

The large quantity of clinical data being generated, wide spectrum of disease presentations and rapidly changing clinical landscape present a critical need for comprehensive evidence summaries and treatment comparisons. The overall aim of this systematic literature review was to assess available evidence regarding efficacy and safety of potential pharmacological interventions for COVID-19. Evidence retrieved was considered in the context of the evolving understanding of the pathophysiology of COVID-19, the burden of COVID-19 in terms of mortality and healthcare resource utilisation, and current knowledge gaps. Thus, the aim of the analysis presented here was to synthesise evidence for mortality, hospitalisation and need for ventilation with current therapies.

\section{Methods}

Overview

This systematic literature review (registered with the Research Registry, unique identifying number: reviewregistry1019) evaluated studies of pharmacological options for the treatment and prevention of COVID-19. The review was conducted according to the principles embodied in the Cochrane Handbook for Systematic Reviews of Interventions [24] and guidance published by the Centre for Reviews and Dissemination [25].

\section{Search strategy}

The systematic literature search was conducted from 1 December 2019 to 14 July 2020 and updated on 14 October 2020, using the electronic databases Embase, MEDLINE via the PubMed platform and the Cochrane Library. Details of the search strings used for each database are presented in supplementary file 1 . Searches were supplemented by reviews of reports of pharmacological interventions for COVID-19 included in a systematic and living map of COVID-19 evidence [26]. These articles were assessed according to the same eligibility criteria as for the systematic searches. As part of the update on 14 October 2020, an additional search of PubMed Central was conducted following retrieval of one article from the systematic and living map that was available in PubMed Central but not in the other databases searched.

\section{Eligibility criteria}

Key inclusion criteria were clinical trials of any pharmacological preventive or treatment approach for COVID-19 of any stage, conducted in human subjects of any age.

Exclusion criteria included: studies of non-pharmacological interventions; traditional or herbal medicines; studies that reported on in vitro or in silico investigations; guidelines; clinical trial protocols or projection studies; or observational studies, such as prospective and retrospective cohort studies, case-control studies, cross-sectional studies, case reports and case series. Articles that were not written in English, reviews, 


\begin{tabular}{|c|c|c|}
\hline Regulatory body & Intervention & Details of approval \\
\hline \multirow[t]{5}{*}{ EMA } & $\begin{array}{l}\text { Pfizer-BioNTech COVID-19 vaccine } \\
\end{array}$ & Conditional marketing authorisation for the prevention of COVID-19 in people $\geqslant 16$ years of age [10] \\
\hline & Moderna COVID-19 vaccine & Conditional marketing authorisation for the prevention of COVID-19 in people $\geqq 18$ years of age [10] \\
\hline & AstraZeneca COVID-19 vaccine & Conditional marketing authorisation for the prevention of COVID-19 in people $\triangleq 18$ years of age [10] \\
\hline & Remdesivir & $\begin{array}{l}\text { Conditional marketing authorisation for the treatment of COVID-19 in adults and adolescents } \geqslant 12 \text { years of age (weighing } \geqslant 40 \\
\mathrm{~kg} \text { ) with pneumonia who require supplemental oxygen [10] }\end{array}$ \\
\hline & Dexamethasone & $\begin{array}{l}\text { Endorsed by the EMA's human medicines committee to treat adults and adolescents } \geqslant 12 \text { years of age (weighing } \geqslant 40 \mathrm{~kg} \text { ) with } \\
\text { COVID- } 19 \text { who require oxygen therapy }[10]\end{array}$ \\
\hline \multirow[t]{7}{*}{ US FDA } & Pfizer-BioNTech COVID-19 vaccine & Emergency use authorisation for the prevention of COVID-19 in people $\geqslant 16$ years of age [11] \\
\hline & Moderna COVID-19 vaccine & Emergency use authorisation for the prevention of COVID-19 in people $\geqslant 18$ years of age [11] \\
\hline & Remdesivir & $\begin{array}{l}\text { Approval for the treatment of adult and paediatric patients } \geqslant 12 \text { years of age (weighing } \geqslant 40 \mathrm{~kg} \text { ) with COVID-19 requiring } \\
\text { hospitalisation. Emergency use authorisation for hospitalised paediatric patients }<12 \text { years of age weighing } \geqslant 3.5 \mathrm{~kg}[11]\end{array}$ \\
\hline & Convalescent plasma & Emergency use authorisation for the treatment of hospitalised patients with COVID-19 [11] \\
\hline & Bamlanivimab & $\begin{array}{l}\text { Emergency use authorisation for the treatment of mild-to-moderate COVID- } 19 \text { in adult and paediatric patients } \geqslant 12 \text { years of } \\
\text { age (weighing } \geqslant 40 \mathrm{~kg} \text { ) with a positive test for SARS-CoV- } 2 \text { and who are at high risk for progressing to severe COVID-19 and/or } \\
\text { hospitalisation [11] }\end{array}$ \\
\hline & $\begin{array}{l}\text { Baricitinib in combination with } \\
\text { remdesivir }\end{array}$ & $\begin{array}{l}\text { Emergency use authorisation for the treatment of suspected or laboratory-confirmed COVID-19 in hospitalised adults and } \\
\text { paediatric patients } \geqslant 2 \text { years of age requiring supplemental oxygen, invasive mechanical ventilation or ECMO [11] }\end{array}$ \\
\hline & Casirivimab and imdevimab & $\begin{array}{l}\text { Emergency use authorisation for the treatment of mild-to-moderate COVID-19 in adults and paediatric patients ( } \geqslant 12 \text { years } \\
\text { weighing at least } 40 \mathrm{~kg} \text { ) with positive results of direct SARS-CoV-2 viral testing, and who are at high risk for progressing to } \\
\text { severe COVID-19 and/or hospitalisation [11] }\end{array}$ \\
\hline
\end{tabular}

b)

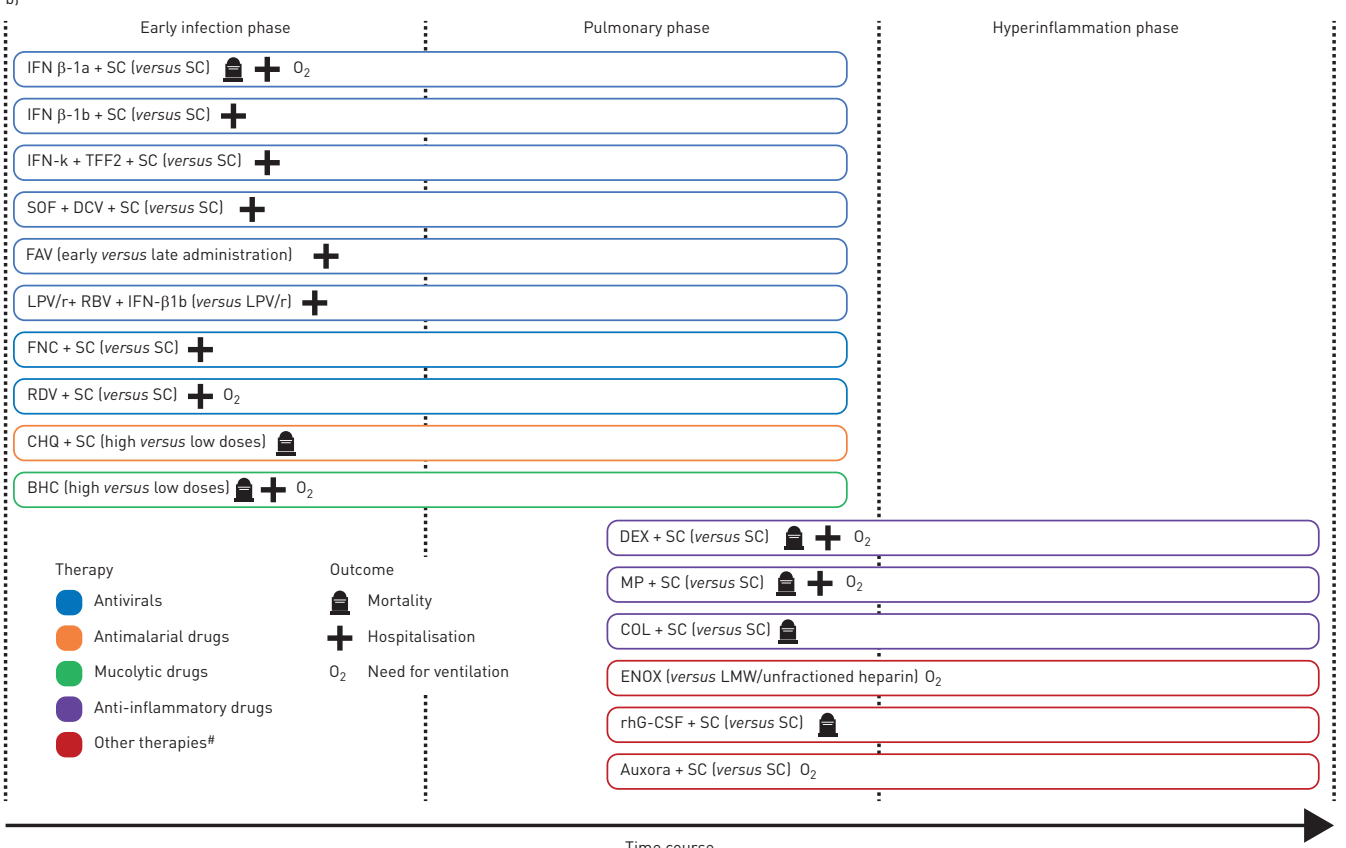

FIGURE 1 a) Current US Food and Drug Administration (FDA) and European Medicines Agency (EMA) approved interventions for coronavirus disease 2019 (COVID-19). b) Effective therapies in the included trials showing interventions that showed statistically significant differences in outcomes versus the comparator. SARS-CoV-2: severe acute respiratory syndrome coronavirus 2; ECMO: extracorporeal membrane oxygenation; IFN: interferon; SC: standard care; TFF2: trefoil factor 2; SOF: sofosbuvir; DCV: daclatasvir; FAV: favipiravir; LPV/r: lopinavir/ritonavir; RBV: ribavirin; FNC: azvudine; RDV: remdesivir; CHQ: chloroquine diphosphate; BHC: bromhexine; DEX: dexamethasone; MP: methylprednisolone; COL: colchicine; ENOX: enoxaparin; LMW: low molecular weight; rhG-CSF: recombinant human granulocyte colony-stimulating factor. \#: includes enoxaparin (anticoagulant), rhG-CSF and auxora (calcium release-activated calcium channel inhibitor).

comments, editorials, congress abstracts, and articles that had not undergone peer review were also excluded.

\section{Study selection}

After removal of duplicates, records identified in the electronic database searches were manually screened for eligibility on the basis of titles and abstracts by a single reviewer, with a second reviewer screening $\geqslant 10 \%$ of records selected at random. Any disagreements or uncertainties were discussed with a third reviewer to achieve a consensus. Subsequently, full texts of potentially relevant studies were obtained and reviewed for eligibility as for the first-pass screening. Studies containing duplicate information or not meeting the inclusion criteria upon further review were excluded. 


\section{Data extraction}

Details of study design and population, interventions, comparators, follow-up duration and safety and efficacy outcomes were extracted from identified articles using a pilot-tested data extraction spreadsheet constructed in Microsoft Excel. If available, information on statistical comparisons between interventions was recorded; any studies reporting insufficient data meeting the inclusion criteria were excluded at the data extraction stage. All extracted data were cross-checked by an independent reviewer.

\section{Data synthesis and quality assessment}

Included studies were reviewed and assessed for comparability in terms of study design and outcomes reported. Randomised studies were included in the qualitative synthesis if they reported $\geqslant 1$ of the selected outcomes: mortality, hospitalisation (any reported outcome, including duration, proportion of patients discharged and incidence) and need for ventilation (use of oxygen, noninvasive ventilation and intensive mechanical ventilation). Non-randomised trials reporting these outcomes were only included if no randomised trial evidence was available for a particular intervention.

Studies were grouped according to intervention and the disease phase targeted by the intervention; details of the categorisation used are presented in supplementary table 1. Studies reporting similar outcome measures were summarised descriptively according to the type of intervention. Studies with outcomes that were defined differently to other studies (e.g. event-free survival rather than percentage mortality, or outcomes reported for the overall population rather than according to treatment with (or allocation to) specific interventions) could not be grouped, and these were assessed separately.

Each study was assessed in terms of methodological quality based on criteria consistent with those in the Cochrane Handbook for Systematic Reviews of Interventions [24] and the Cochrane risk-of-bias tool [24, 27].

\section{Results}

\section{Summary of included studies}

The literature searches identified 33674 unique citations, and from these, 436 full-text articles were assessed for eligibility (supplementary figure 1). A total of 375 articles were excluded as they did not meet eligibility criteria for reasons such as reporting no outcomes of interest, presenting duplicate data from other studies or having a non-interventional study design. A total of 61 articles were retained for inclusion in the systematic literature review and, of these, 43 articles, reporting on 42 trials [28-70], were selected for qualitative synthesis, based on whether they reported $\geqslant 1$ of the outcomes of interest.

Details of the 18 articles that were not retained for qualitative synthesis are summarised in supplementary table 2. An overview of the 43 articles included in the qualitative synthesis is presented in supplementary figure 2, and detailed characteristics of the individual trials are presented in supplementary table 3 . The majority of trials were conducted in patients hospitalised with COVID-19, but three trials included non-hospitalised patients $[35,40,42]$ and one was conducted in asymptomatic adults with occupational or household exposure to COVID-19 [30] (supplementary figure 2). Most trials used either standard care, which differed between trials, or placebo as a comparator.

Significant findings across all outcomes assessed are summarised in figure $1 \mathrm{~b}$, with a detailed description of all findings in the following sections.

\section{Mortality}

In total, 39 randomised and one non-randomised trial reported on mortality, either as the number of deaths that occurred during the study or as a pre-specified study end-point, with or without a statistical comparison between groups [28-34, 36-54, 56-68, 70]. One study included [69] was a re-analysis of data from another trial (WANG et al. [44]) included in the qualitative synthesis. The outcomes for individual trials are shown in figure 2.

\section{Antivirals}

Among trials of antivirals (figure 2a), four trials $(n=48-66)$ reported trends towards decreased mortality with interferons (IFNs), sofosbuvir+daclatasvir, and triazavirin, compared with standard care or placebo in patients with COVID-19 of varying severity [59,60,67,68]. Another trial $(n=81)$ reported a significant reduction in 28-day mortality with IFN- $\beta$-1a plus standard care compared with standard care alone (19.0\% versus 43.6\%; $\mathrm{p}=0.015$ ) in patients with severe COVID-19 [64]. The analysis also showed that administration of IFN $\beta$-1a early in the disease significantly reduced mortality (OR 13.5 (95\% CI) 1.5-118) whereas late administration did not [64].

Of four trials that assessed lopinavir/ritonavir, three trials of patients with COVID-19 of varying severity $(n=86-127)$ reported no deaths in either treatment group [37, 39, 62]. One trial $(n=199)$ reported a 

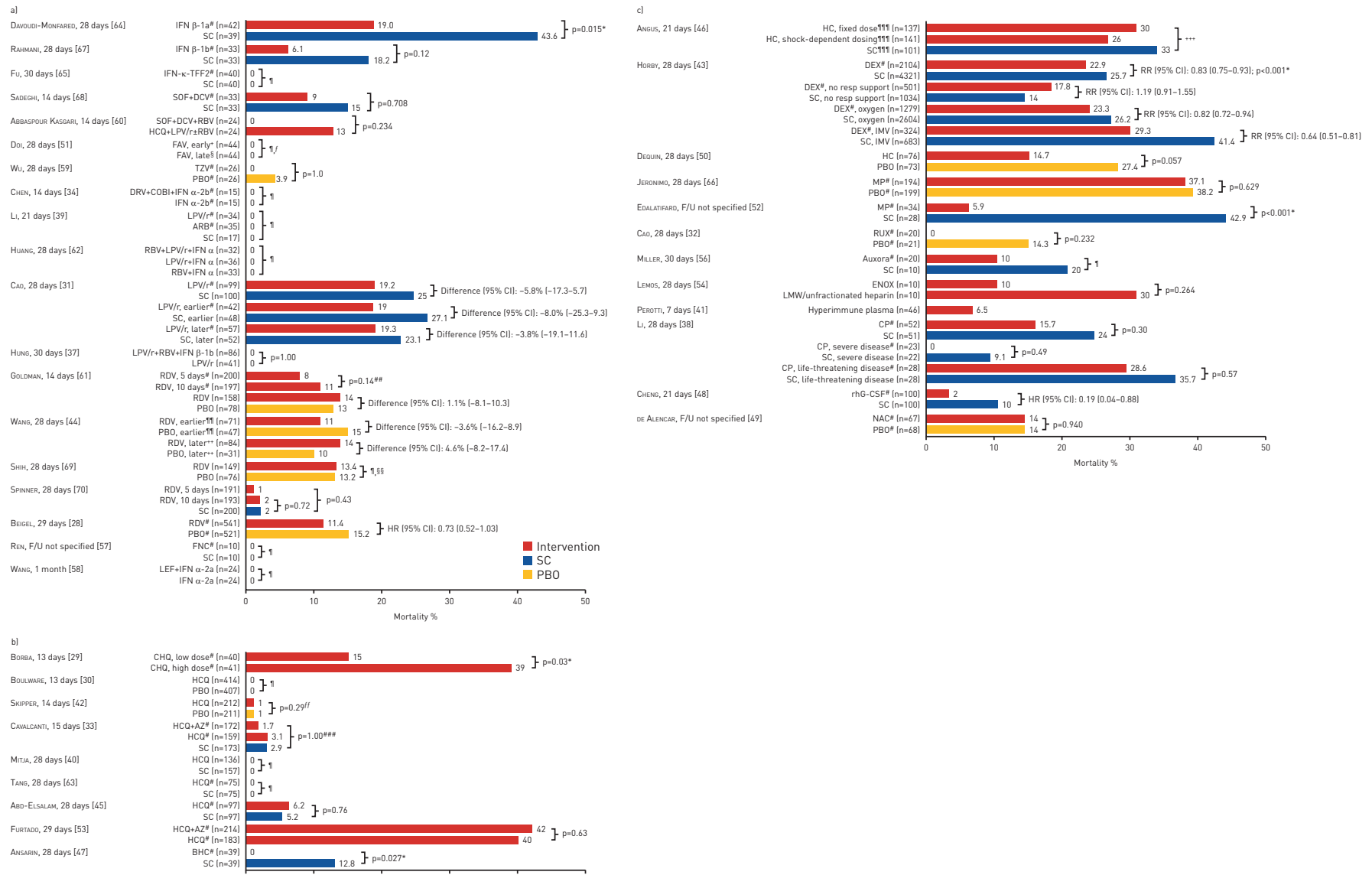

FIGURE 2 Summary of mortality outcomes in trials of a) antivirals, b) antimalarial and mucolytic drugs and c) other therapies included in the qualitative synthesis. Other therapies include anti-inflammatory drugs, anticoagulants, kinase inhibitors, calcium release-activated calcium channel inhibitors, anticoagulants, immunomodulatory therapies and repair therapies. Results from one study are not presented graphically. Deftereos et al. [36] reported event-free survival as a primary outcome, which was defined as survival without meeting the primary clinical end-point (deterioration by 2 points on a 7-grade clinical status scale, ranging from able to resume normal activities to death). IFN: interferon; SC: standard care; TFF2: trefoil factor 2; SOF: sofosbuvir; DCV: daclatasvir; RBV: ribavirin; HCQ: hydroxychloroquine; LPV/r: lopinavir/ritonavir; FAV: favipiravir; TZV: triazavirin; PBO: placebo; DRV: darunavir; COBI: cobicistat; ARB: arbidol; RDV: remdesivir; F/U: follow-up; FNC: azvudine; LEF: leflunomide; CHQ: chloroquine diphosphate; AZ: azithromycin; BHC: bromhexine; HC: hydrocortisone; DEX: dexamethasone; IMV: invasive mechanical ventilation; MP: methylprednisolone; RUX: ruxolitinib; ENOX: enoxaparin; LMW: low molecular weight; CP: convalescent plasma; rhG-CSF: recombinant human granulocyte colony-stimulating factor; NAC: N-acetylcysteine. ${ }^{\#}$ : treatment administered in addition to SC, as defined by the investigators in each trial; ${ }^{\text {I: }}$ no between-group comparison; ${ }^{+}$: treatment on day 1 of study participation; ${ }^{\S}$ : treatment on day 6 of

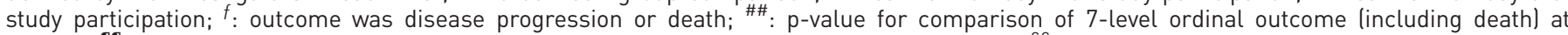
14 days; "१: treatment $\leqslant 10$ days of symptom onset; ${ }^{++}$: treatment $>10$ days of symptom onset; ${ }^{\S \S}$; re-analysis of data from WANG et al. [44] using different criteria; ${ }^{f f}$ : outcome was incidence of hospitalisation or death; ${ }^{\# \# \#}$ : p-value for comparisons of the 7-level ordinal outcome lincluding death) at 15 days; กाก: participants could be randomly assigned to other interventions within other therapeutic domains; ${ }^{+++}:$median ( $95 \%$ Cl) adjusted odds ratios versus the no-hydrocortisone group were 1.03 (0.53-1.95) and 1.10 (0.58-2.11) for the fixed-dose and shock-dependent dosing hydrocortisone groups, respectively. These yielded $54 \%$ and $62 \%$ Bayesian posterior probabilities of superiority. *: statistically significant p-value.

numerical but nonsignificant reduction in 28-day mortality with lopinavir/ritonavir versus standard care in patients with severe COVID-19 [31]. Of four trials that investigated remdesivir in patients with moderate or severe COVID-19, two trials $(\mathrm{n}=1062$ and $\mathrm{n}=236)$ and the re-analysis of WANG et al. [44] by SHIH et al. [69] showed no significant mortality benefit of remdesivir compared with placebo [28, 44], although one study showed a trend towards reduced mortality in patients who received treatment earlier in their disease course (within 10 days of symptom onset) [44]. The other two trials ( $n=397$ and $n=596)$ reported comparable mortality with 5- and 10-day remdesivir treatment $[61,70]$.

\section{Antimalarial and mucolytic drugs}

Among eight trials of hydroxychloroquine or its derivatives (figure 2b), one trial $(\mathrm{n}=81$ ) reported significantly greater lethality with high doses of chloroquine diphosphate compared with low doses (log-rank: -2.183; $\mathrm{p}=0.03)$ in patients with severe COVID-19 [29]. Six trials $(\mathrm{n}=150-821)$ reported similar mortality with hydroxychloroquine, with or without azithromycin, compared with standard care or 
placebo in hospitalised [33, 45, 63] or non-hospitalised [30, 40, 42] patients with mild, mild-to-moderate or severe COVID-19. Another trial $(n=447)$ reported no mortality benefit of adding azithromycin to hydroxychloroquine compared with hydroxychloroquine alone in patients with severe COVID-19 [53]. In a trial of the mucolytic drug, bromhexine $(n=78)$, there was a significant reduction in mortality with bromhexine plus standard care versus standard care alone $(0 \%$ versus $12.8 \% ; \mathrm{p}=0.027)$ in patients with COVID-19 of unspecified severity [47].

\section{Anti-inflammatory drugs}

Among trials of corticosteroids conducted in patients with severe COVID-19, three trials ( $\mathrm{n}=149-403)$ showed numerical but nonsignificant trends towards reduced mortality with hydrocortisone or methylprednisolone compared with placebo or standard care (figure 2c) $[46,50,66]$. One trial $(n=62)$ reported significantly reduced mortality $(5.9 \%$ versus $42.9 \% ; p<0.001)$ with methylprednisolone plus standard care versus standard care alone [52] (figure 2c). In a large trial $(n=6425)$ of patients with COVID-19 of unspecified severity, 28-day mortality was significantly decreased with dexamethasone plus standard care versus standard care alone, both overall $(22.9 \%$ versus $25.7 \% ; p<0.001)$ and in patients receiving oxygen $(23.3 \%$ versus $26.2 \%)$ or mechanical ventilation $(29.3 \%$ versus $41.4 \%)$ at randomisation (figure 2c) [43]. The mortality benefit was greatest in patients with a longer duration of symptoms ( $>7$ days versus $\leqslant 7$ days; 12.3 by Chi-squared test for trend) [43]. Another trial $(\mathrm{n}=105)$, also conducted in patients with COVID-19 of unspecified severity, showed significantly increased event-free survival with the anti-inflammatory drug colchicine in combination with standard care versus standard care alone $(97 \%$ versus $83 \%$ of patients after 10 days; $\mathrm{p}=0.03$; data not shown graphically) [36].

\section{Other therapies}

Trials $(n=20-135)$ investigating the kinase inhibitor, ruxolitinib [32], the calcium release-activated calcium channel inhibitor, auxora [56], the anticoagulant, enoxaparin [54], and N-acetylcysteine, a mucolytic drug with anti-oxidant properties [49], in patients with severe COVID-19 reported no significant difference in mortality versus the comparator groups (figure $2 \mathrm{c})$. One trial $(\mathrm{n}=200)$ reported a reduced 21-day mortality with recombinant human granulocyte colony-stimulating factor (rhG-CSF) added to standard care versus standard care alone (HR 0.19 (95\% CI 0.04-0.88)) in patients with severe COVID-19 [48].

Two studies reported on immunomodulatory therapies in patients with moderate and/or severe COVID-19 (figure $2 \mathrm{c}$ ): one single-arm trial $(\mathrm{n}=46)$ reported a mortality of $6.5 \%$ with hyperimmune plasma [41], and another trial $(\mathrm{n}=103)$ reported numerical but nonsignificant trends towards decreased mortality with convalescent plasma versus standard care [38].

\section{Hospitalisation}

In total, 36 randomised trials and one non-randomised trial reported on hospitalisation, either as a pre-specified study end-point or the number of patients experiencing a particular outcome during the study, with or without a statistical comparison between groups $[28,30-33,35-38,40-55,57-62,64-68$, 70]. Additionally, one study included [69] was a re-analysis of data from another trial (WANG et al. [44]) included in the qualitative synthesis. Outcomes reported across studies included median duration of hospitalisation (figure 3), proportion of patients discharged during the study (table 1), incidence of intensive care unit (ICU) admittance (table 1) and incidence of hospitalisation (supplementary figure 3 ).

\section{Antivirals}

Three trials of IFNs (IFN- $\beta-1 \mathrm{a}$, IFN- $\beta-1 \mathrm{~b}$ or IFN- $\kappa+$ trefoil factor 2$)(\mathrm{n}=66-81)$ added to standard care in patients with moderate or severe COVID-19 reported improvements in hospitalisation outcomes, including reduced hospitalisation duration [65, 67] (figure 3a), a greater proportion of patients discharged [64, 67] (table 1) and reduced incidence of ICU admittance [64,67] (table 1) versus standard care alone. Other trials $(\mathrm{n}=66$ and $\mathrm{n}=88$ ) reported reduced hospitalisation duration with sofosbuvir+daclatasvir+standard care ( 6 versus 8 days; $\mathrm{p}=0.029)$ and early versus late administration of favipiravir (14.5 versus 20 days; HR 1.963 (95\% CI 1.331-2.894)) in patients with moderate or severe COVID-19 [68] or asymptomatic to mild COVID-19 [51], respectively (figure 3a). Patients treated with sofosbuvir+daclatasvir+standard care also had a significantly higher probability of hospital discharge by day $14(\mathrm{p}=0.041)$ versus standard care alone (table 1).

Another trial $(\mathrm{n}=127)$, conducted in patients with COVID-19 of unspecified severity, reported a significant reduction in median hospitalisation duration with lopinavir/ritonavir+ribavirin+IFN- $\beta-1 \mathrm{~b}$ versus lopinavir/ ritonavir alone [37]. When patients were stratified according to the timing of treatment administration, median hospitalisation was significantly reduced in patients who received treatment within 7 days of symptom onset, but not in those who received treatment later than this [37]. 
a)

DAVOUdI-MONFARED\# [64]

RAHMANI, [67]

$\mathrm{Fu}^{+}$[65]

SADEGHI [68]

AbBaspour KasgarI [60]

Doıई [51]

CAO [31]

HuAng [62]

HUNG [37]

Beigel [28]

WANG [44]

GoLdMAN [61]

REN\# [57]

WANG [58]

Abd-ElSALAM\# [45]

FurTADoff [53]

Cavalcanti \# [33]

b)

HoRBY [43]

JeRonimo [66]

Deftereos [36]

Lemos [54]

LI [38]

Cheng [48]

DE Alencar [49]
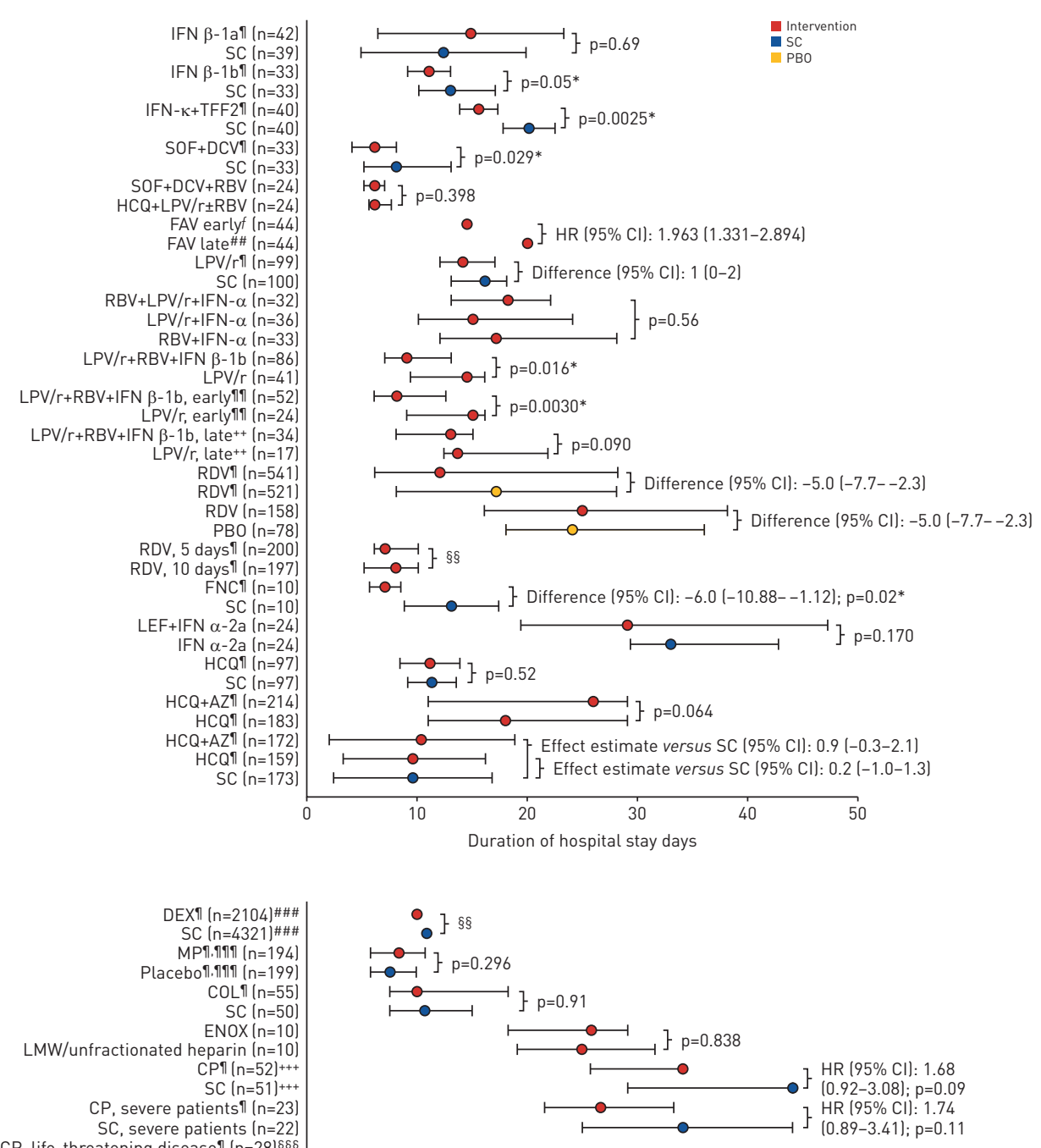

$C P$, life-threatening disease? $(n=28) \S \S \S$ $S C$, life-threatening disease $(n=28) \S \S \S$ rhG-CSFף $(n=100)$ NAC? $(n=67)$ SC $[n=68$

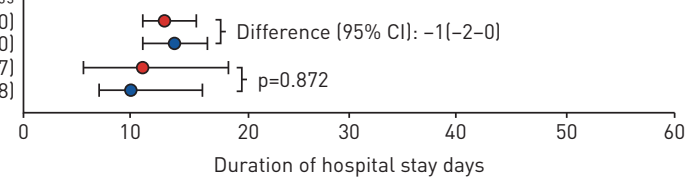

FIGURE 3 Duration of hospitalisation in trials of a) antivirals, antimalarial and mucolytic drugs and b) other therapies included in the qualitative synthesis. Data are presented as median (IQR) unless indicated otherwise. Other therapies include anti-inflammatory drugs, anticoagulants, immunomodulatory therapies and repair therapies. IFN: interferon; SC: standard care; TFF2: trefoil factor 2; SOF: sofosbuvir; DCV: daclatasvir; RBV: ribavirin; HCQ: hydroxychloroquine; LPV/r: lopinavir/ritonavir; FAV: favipiravir; RDV: remdesivir; PBO: placebo; FNC: azvudine; LEF: leflunomide; AZ: azithromycin; DEX: dexamethasone; MP: methylprednisolone; COL: colchicine; ENOX: enoxaparin; LMW: low molecular weight; CP: convalescent plasma; rhG-CSF: recombinant human granulocyte colony-stimulating factor; NAC: N-acetylcysteine. " : mean \pm SD; ${ }^{\text {": }}$ treatment administered in addition to SC, as defined by the investigators in each trial; ${ }^{+}$: mean $(95 \% \mathrm{Cl}) ;{ }^{\S}$ : post-hoc analysis; ${ }^{f}$ : treatment on day 1 of study

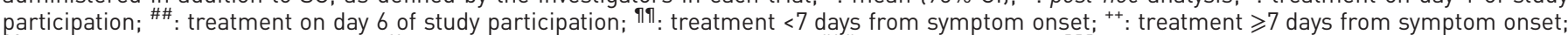

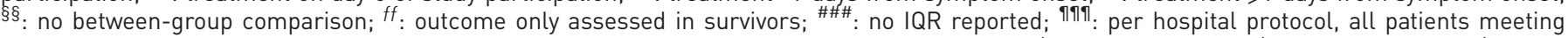
acute respiratory distress syndrome criteria were given pre-emptively intravenous ceftriaxone $(1 \mathrm{~g}$ twice for 7 days) plus azithromycin (500 mg once for 5 days) or clarithromycin (500 mg twice for 7 days), starting on day $1 ;^{+++}$: upper IQR limit could not be determined; ${ }^{\S \S \S}$ : median value could not be determined, HR $1.90(95 \% \mathrm{Cl} 0.45-8.04) ; p=0.38$. *: statistically significant $p$-value.

Among trials of remdesivir and the re-analysis of WANG et al. [44], one trial ( $\mathrm{n}=1062)$ reported a reduced initial length of hospital stay with remdesivir versus placebo in patients with severe COVID-19 (median 12 versus 17 days) [28] (figure 3a). There were trends towards more patients discharged with remdesivir versus placebo/standard care, as well as with earlier remdesivir treatment in the remaining trials [44, 61, 69, 70] (table 1), but between-group differences were either not significant or not tested. A small pilot study $(\mathrm{n}=20)$ also reported a reduced mean duration of hospitalisation ( 7 versus 13 days; $\mathrm{p}=0.02)$ with the antiretroviral azvudine plus standard care versus standard care alone in patients with mild COVID-19 [57]. 
TABLE 1 Summary of hospitalisation outcomes in trials included in the qualitative synthesis

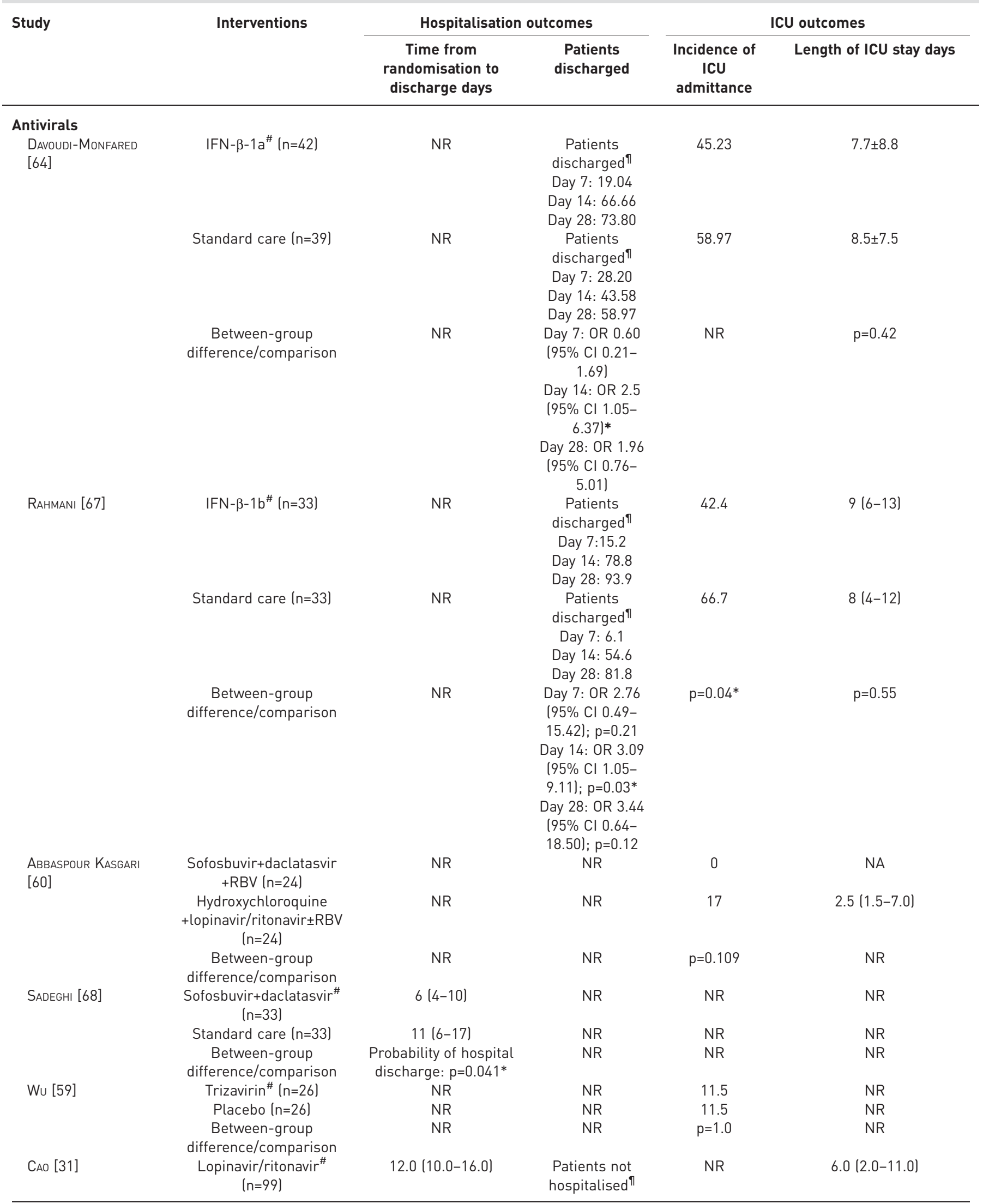




\section{TABLE 1 Continued}

\begin{tabular}{|c|c|c|c|c|c|}
\hline \multirow[t]{2}{*}{ Study } & \multirow[t]{2}{*}{ Interventions } & \multicolumn{2}{|c|}{ Hospitalisation outcomes } & \multicolumn{2}{|r|}{ ICU outcomes } \\
\hline & & $\begin{array}{l}\text { Time from } \\
\text { randomisation to } \\
\text { discharge days }\end{array}$ & $\begin{array}{c}\text { Patients } \\
\text { discharged }\end{array}$ & $\begin{array}{l}\text { Incidence of } \\
\text { ICU } \\
\text { admittance }\end{array}$ & Length of ICU stay days \\
\hline & & & $\begin{array}{c}\text { Day } 7: 4 \\
\text { Day 14: } 43.4\end{array}$ & & \\
\hline & Standard care $(n=100)$ & $14.0(11.0-16.0)$ & $\begin{array}{l}\text { Patients not } \\
\text { hospitalised } \\
\text { Day 7: } 0 \\
\text { Day 14: } 28\end{array}$ & NR & $11.0(7.0-17.0)$ \\
\hline & $\begin{array}{c}\text { Between-group } \\
\text { difference/comparison }\end{array}$ & $\begin{array}{c}\text { Difference } 1.0(95 \% \mathrm{Cl} \\
0-3.0)\end{array}$ & NR & NR & Difference $-5(95 \% \mathrm{Cl}-9-0)$ \\
\hline \multirow[t]{2}{*}{ HUNG [37] } & $\begin{array}{l}\text { Lopinavir/ritonavir+RBV } \\
+ \text { IFN- } \beta-1 b \quad(n=86)\end{array}$ & NR & NR & Overall: 5 & NR \\
\hline & Lopinavir/ritonavir ( $n=41$ ) & NR & NR & & NR \\
\hline \multirow{4}{*}{ GoLdMAN [61] } & $\begin{array}{l}\text { Remdesivir, 10-day } \\
\text { treatment }{ }^{\#}(n=197)\end{array}$ & NR & $\begin{array}{l}\text { Within } 14 \text { days: } \\
52\end{array}$ & NR & NR \\
\hline & $\begin{array}{l}\text { Overall, symptoms } \\
\quad<10 \text { days } \#\end{array}$ & NR & $\begin{array}{l}\text { Within } 14 \text { days: } \\
62\end{array}$ & NR & NR \\
\hline & $\begin{array}{l}\text { Overall, symptoms } \\
>10 \text { days }{ }^{\#}\end{array}$ & NR & $\begin{array}{l}\text { Within } 14 \text { days: } \\
49\end{array}$ & NR & NR \\
\hline & $\begin{array}{c}\text { Between-group } \\
\text { difference/comparison }\end{array}$ & NR & NR & NR & NR \\
\hline WANG [44] & Remdesivir (n=158) & $21.0(12.0-31.0)$ & $\begin{array}{c}\text { Patients } \\
\text { discharged } \\
\text { (alive)" } \\
\text { Day 14: } 25 \\
\text { Day 28: } 61\end{array}$ & NR & NR \\
\hline \multirow[t]{3}{*}{$\mathrm{SHIH}^{+}$[69] } & Remdesivir ( $n=158$ ) & NR & $\begin{array}{c}\text { Day } 14(n=151): \\
29.8 \\
\text { Day } 28(n=149): \\
66.4\end{array}$ & NR & NR \\
\hline & Placebo (n=78) & NR & $\begin{array}{c}\text { Day } 14(\mathrm{n}=78) \text { : } \\
23.1 \\
\text { Day } 28(\mathrm{n}=78) \text { : } \\
60.5\end{array}$ & NR & NR \\
\hline & $\begin{array}{c}\text { Between-group } \\
\text { difference/comparison }\end{array}$ & NR & NR & NR & NR \\
\hline \multirow[t]{2}{*}{ SPINNER [70] } & $\begin{array}{l}\text { Remdesivir, 5-day } \\
\text { treatment (n=191) }\end{array}$ & NR & $\begin{array}{l}\text { Patients not } \\
\text { hospitalised } \\
\text { Day 11:70 } \\
\text { Day 14: } 76 \\
\text { Day 28: } 89\end{array}$ & NR & NR \\
\hline & $\begin{array}{l}\text { Remdesivir, 10-day } \\
\text { treatment ( } n=193 \text { ) }\end{array}$ & NR & $\begin{array}{l}\text { Patients not } \\
\text { hospitalised" } \\
\text { Day 11: } 65\end{array}$ & NR & NR \\
\hline
\end{tabular}




\section{TABLE 1 Continued}

\section{Study}

Interventions

Standard care $(n=200)$

Between-group difference/comparison

\section{Antimalarial drugs}

Abd-Elsalam [45]

\section{Mucolytic drugs \\ ANSARIN [47] \\ LI [55] \\ Anti-inflammatory drugs}

Angus [46]

HoRBY [43]

Dequin [50]

EDALATIFARD [52]
Hydroxychloroquine ${ }^{\#}$ ( $n=97)$

Standard care $(n=97)$

Between-group

difference/comparison

$$
\begin{gathered}
\text { Bromhexine }^{\#}(\mathrm{n}=39) \\
\text { Standard care ( } \mathrm{n}=39) \\
\text { Between-group }
\end{gathered}
$$$$
\text { difference/comparison }
$$$$
\text { Bromhexine }{ }^{\#}(n=12)
$$

Standard care $(n=6)$

Between-group difference/comparison

$$
\text { Hydrocortisone, fixed }
$$$$
\text { dose } \left.^{\S} \text { ( } n=137\right)
$$

Hydrocortisone, shock-dependent dosing ${ }^{\S}$ ( $n=141)$

Standard care $^{\S}$ (n=101)

Between-group difference/comparison

$$
\begin{gathered}
\text { Dexamethasone } \\
\quad(n=2104)
\end{gathered}
$$

Standard care $(n=4321)$ difference/comparison Hydrocortisone ( $n=76$ )

Placebo $(n=73)$

Between-group difference/comparison Methylprednisolone ${ }^{\#}$ ( $n=34$ )

Standard care $(n=28)$
Between-group
Hospitalisation outcomes

$\begin{array}{cc}\text { Time from } & \begin{array}{c}\text { Patients } \\ \text { discharged }\end{array}\end{array}$

discharge days
Day 14: 76

Day 28: 90

Patients not hospitalised"

Day 11: 60

Day 14: 67

Day 28: 83

NR

NR

NR

NR

$7.6 \pm 3.5$

$8.1 \pm 5.5$

$p=0.587$

NR

NR

NR

NR

NR

NR

NR

NR

NR

NR

NR

NR

NR

Time to discharge/ death: $11.62 \pm 4.81$

NR

NR

NR

NR

83.3

33.3

$\mathrm{p}=0.12$

NR

NR

NR

$$
67.2
$$

$$
63.5
$$
1.03-1.17)* ICU $\$$ : 57.3 ICU ${ }^{\S}: 43.8$

$\mathrm{NR}$

NR
ICU outcomes

Incidence of Length of ICU stay days

ICU

admittance
NR

NR

NR

NR

11.3

NR

13.4

NR

$\mathrm{p}=0.83$

NR

Within 20 days:

Within 20 days:

NR

\section{1 \\ 28.2}

$p=0.006^{*}$

NR

NR

NR

NR

NR

NR

NR

NR

NR

$N R$

NR

NR

Within 28 days:

NR

Within 28 days:

RR $1.10(95 \% \mathrm{Cl}$

Discharge from

Discharge from

NR

Adjusted HR:

$0.93 \pm 0.14$

Median 0.92 (95\% Cl 0.681.24)

Adjusted HR:

$0.86 \pm 0.13$

Median 0.85 (95\% Cl 0.62-

1.15)

Mean: 1 (Reference)

Median: 1 (Reference)

Probability of superiority to standard care, $29 \%$ (fixed dose) and $14 \%$

(shock-dependent dosing)

NR

NR

NR

NR

NR

NR

NR

NR

NR

NR

NR

NR

NR

NR

NR 


\section{TABLE 1 Continued}

\begin{tabular}{|c|c|c|c|c|c|}
\hline \multirow[t]{2}{*}{ Study } & \multirow[t]{2}{*}{ Interventions } & \multicolumn{2}{|c|}{ Hospitalisation outcomes } & \multicolumn{2}{|c|}{ ICU outcomes } \\
\hline & & $\begin{array}{l}\text { Time from } \\
\text { randomisation to } \\
\text { discharge days }\end{array}$ & $\begin{array}{l}\text { Patients } \\
\text { discharged }\end{array}$ & $\begin{array}{l}\text { Incidence of } \\
\text { ICU } \\
\text { admittance }\end{array}$ & Length of ICU stay days \\
\hline \multirow{4}{*}{ DavoodI [35] } & & $\begin{array}{l}\text { Time to discharge/ } \\
\text { death: } 17.61 \pm 9.84\end{array}$ & & & \\
\hline & $\begin{array}{c}\text { Between-group } \\
\text { difference/comparison }\end{array}$ & $p=0.006^{*}$ & NR & NR & NR \\
\hline & $\begin{array}{l}\text { Hydroxychloroquine } \\
\qquad(n=25)\end{array}$ & NR & $\begin{array}{l}100 \text { (of three } \\
\text { patients } \\
\text { hospitalised) }\end{array}$ & 0 & NR \\
\hline & $\begin{array}{c}\text { Between-group } \\
\text { difference/comparison }\end{array}$ & NR & NR & NR & NR \\
\hline & $\begin{array}{c}\text { Between-group } \\
\text { difference/comparison }\end{array}$ & $p=0.941$ & NR & NR & NR \\
\hline \multicolumn{6}{|c|}{$\begin{array}{l}\text { Immunomodulatory } \\
\text { therapies }\end{array}$} \\
\hline PerotTI [41] & $\begin{array}{l}\text { Hyperimmune plasma } \\
\qquad(\mathrm{n}=46)\end{array}$ & NR & Within 7 days: 0 & NR & NR \\
\hline \multirow[t]{4}{*}{ LI [38] } & Standard care $(n=51)$ & $\begin{array}{l}\text { Indeterminate (19.0- } \\
\text { indeterminate) } \\
\text { Time from } \\
\text { hospitalisation to } \\
\text { discharge: } 53.0 \text { (35.0- } \\
\text { indeterminate) }\end{array}$ & $\begin{array}{l}\text { Within } 28 \text { days: } \\
\quad 36.0\end{array}$ & NR & NR \\
\hline & $\begin{array}{c}\text { Standard care, severe } \\
\text { disease }(n=51)\end{array}$ & $\begin{array}{c}19.0(11.0- \\
\text { indeterminate) } \\
\text { Time from } \\
\text { hospitalisation to } \\
\text { discharge: } 41.0 \text { (30.0- } \\
53.0 \text { ) }\end{array}$ & $\begin{array}{l}\text { Within } 28 \text { days: } \\
\quad 68.2\end{array}$ & NR & NR \\
\hline & $\begin{array}{l}\text { Convalescent plasma, } \\
\text { severe disease }(n=23)\end{array}$ & $\begin{array}{c}13.0(10.0-16.0) \\
\text { Time from } \\
\text { hospitalisation to } \\
\text { discharge: } 32.0 \text { (26.0- } \\
40.0)\end{array}$ & $\begin{array}{l}\text { Within } 28 \text { days: } \\
\quad 91.3\end{array}$ & NR & NR \\
\hline & $\begin{array}{c}\text { Between-group } \\
\text { difference/comparison }\end{array}$ & $\begin{array}{c}\text { HR } 1.97(95 \% \mathrm{Cl} 1.00- \\
\text { 3.88); } p=0.05 \\
\text { Hospitalisation to } \\
\text { discharge: HR } 1.74 \\
(95 \% \mathrm{Cl} 0.89-3.41) ; \\
\mathrm{p}=0.11\end{array}$ & $\begin{array}{c}\text { OR } 4.90(95 \% \mathrm{Cl} \\
0.89-26.97) \\
p=0.07\end{array}$ & NR & NR \\
\hline
\end{tabular}


TABLE 1 Continued

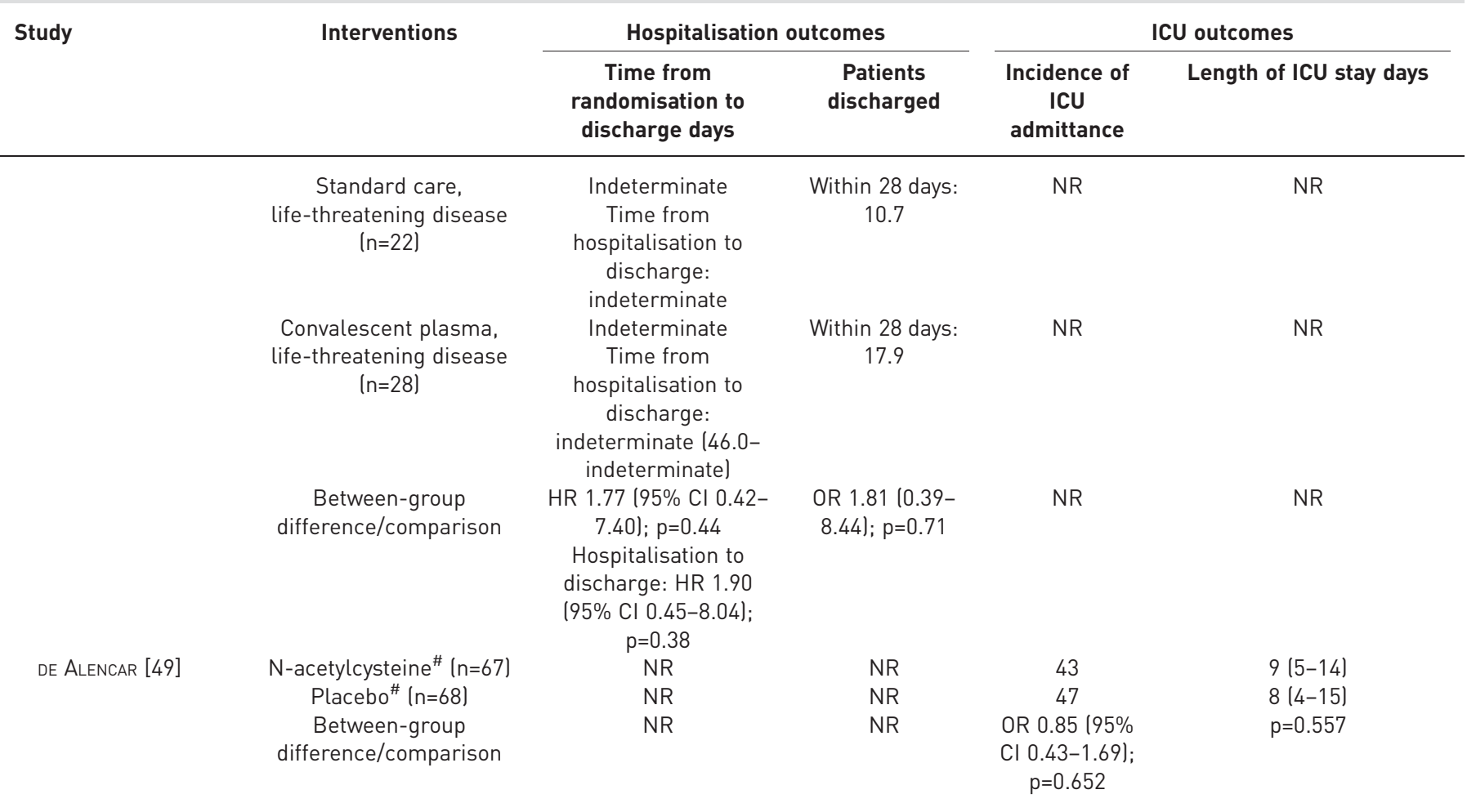

Data are presented as median (interquartile range), \% or mean \pm SD, unless otherwise stated. ICU: intensive care unit; IFN: interferon; NR: not reported; NA: not applicable; RBV: ribavirin. " : treatment administered in addition to standard care, as defined by the investigators in each trial; " : outcome was reported as part of a modified 6- or 7-category ordinal scale of clinical status; ${ }^{+}$: re-analysis of data from WANG et al. [44], using different criteria; ${ }^{\S}$ : participants could be randomly assigned to other interventions within other therapeutic domains. *: statistically significant $\mathrm{p}$-value or between-group comparison.

\section{Antimalarial and mucolytic drugs}

Among six trials assessing hydroxychloroquine $(\mathrm{n}=194-821)$ [30, 33, 40, 42, 45, 53], with or without azithromycin, no benefit was seen relative to the comparator groups in terms of hospitalisation duration (figure 3a), incidence of ICU admittance (table 1) or incidence of hospitalisation (supplementary figure 3). In a trial conducted in patients with COVID-19 of unspecified severity $(n=78)$, ICU admittance was significantly reduced with bromhexine plus standard care versus standard care alone $(5.1 \%$ versus $28.2 \%$; $\mathrm{p}=0.006$ ) [47] (table 1).

\section{Anti-inflammatory drugs}

One trial $(n=6425)$ reported a numerically shorter median duration of hospitalisation (12 versus 13 days) and a greater probability of discharge alive within 28 days with dexamethasone plus standard care versus standard care alone (rate ratio 1.10 (95\% CI 1.03-1.17)) in patients with COVID-19 of unspecified severity [43] (figure 3b and table 1). In another trial of patients with severe COVID-19 ( $\mathrm{n}=403)$, treatment with a 7-day fixed-dose course or shock-dependent dosing of hydrocortisone were associated with reduced hazard ratios for length of hospital and ICU stay; however, neither treatment strategy met pre-specified criteria for statistical superiority [46]. A further trial $(n=62)$ reported a significantly reduced time to the composite outcome of hospital discharge or death with methylprednisolone plus standard care versus standard care alone in patients with severe COVID-19 (median 11.6 versus 17.6 days; $p=0.006$ ) [52] (table 1). Other trials of anti-inflammatory agents $(n=54-416)$ reported no differences in hospitalisation outcomes versus comparators (figure $3 \mathrm{~b}$, table 1 and supplementary figure 3 ) $[35,36,50,66]$.

\section{Other therapies}

Among trials of other therapies, one trial $(n=103)$ reported numerical but nonsignificant trends towards reduced hospitalisation duration and increased numbers of patients discharged with convalescent plasma versus standard care [38] (figure $3 b$ and table 1). Trials of rhG-CSF $(n=200), N$-acetylcysteine $(n=135)$, 
enoxaparin $(n=20)$ and ruxolitinib $(n=43)$ reported no significant impact of these interventions on hospitalisation outcomes versus standard care or placebo [32, 48, 49, 54] (figure 3b and table 1).

\section{Need for ventilation}

In total, 30 randomised and one non-randomised trial, and one re-analysis of the study by WANG et al. [44], reported outcomes relating to the need for oxygen, noninvasive ventilation or intensive mechanical ventilation $[28,31-34,36,37,39-41,43-50,52-56,59-61,64,66-70]$. These end-points were reported either as pre-specified study end-points or the number of patients receiving a particular intervention during the study, with or without a statistical comparison between groups. The outcomes for individual trials are shown in figure 4 and supplementary table 4.

\section{Antivirals}

Most trials of antivirals did not report a significant impact of the interventions assessed on the number of patients requiring ventilation, or on related outcomes including duration of respiratory support (figure $4 \mathrm{a}$ and supplementary table 4). However, there were trends towards decreased use of ventilation with IFN therapies, sofosbuvir+daclatasvir and triazavirin (figure 4a) $[59,60,64,68]$. Additionally, one trial $(\mathrm{n}=81$ ) of patients with severe COVID-19 reported an increased number of patients extubated following treatment with IFN- $\beta$-1a plus standard care than with standard care alone (53.5\% versus $11.8 \%$; $\mathrm{p}=0.019)$ [64] (supplementary table 4). Another trial $(\mathrm{n}=1062)$, also conducted in patients with severe COVID-19, reported fewer patients requiring new use of oxygen (36\% versus $44 \%$ ), noninvasive ventilation ( $17 \%$ versus $24 \%$ ) or intensive mechanical ventilation (13\% versus $23 \%$ ) with remdesivir versus placebo (figure $4 \mathrm{a})$ [28].

\section{Antimalarial and mucolytic drugs}

Four trials ( $n=194-665)$ reported no significant impact of hydroxychloroquine, with or without azithromycin, on reducing the need for ventilation or improving other respiratory outcomes compared with standard care, in patients with COVID-19 of a range of severities [33, 40, 45, 53] (figure $4 \mathrm{~b}$ and supplementary table 4). Among two trials $(n=78$ and $n=18)$ of bromhexine plus standard versus standard care alone, one reported significantly reduced numbers of patients with COVID-19 of unspecified severity requiring intensive mechanical ventilation with bromhexine (2.6\% versus $23.1 \%$; $\mathrm{p}=0.007$ ) [47] (figure $4 \mathrm{~b}$ ). The other trial showed a numerical but nonsignificant trend towards reduced need for oxygen therapy with bromhexine $(16.7 \%$ versus $33.3 \%$; $\mathrm{p}=0.11$ ) versus standard care, in patients with mild or moderate COVID-19 [55] (figure 4b).

\section{Anti-inflammatory drugs}

Among studies of anti-inflammatory drugs (figure $4 \mathrm{~b}$ and supplementary table 4 ), one randomised trial $(n=6425)$ reported a statistically significantly decreased need for invasive mechanical ventilation with dexamethasone plus standard care versus standard care alone in patients with COVID-19 of unspecified severity [43] (figure 4b). The risk of progression to invasive mechanical ventilation was also significantly lower with dexamethasone than with standard care (risk ratio 0.77 (95\% CI 0.62-0.95)) [43]. Another trial $(n=62)$ of patients with severe COVID-19 reported a significant reduction in the proportion of patients receiving oxygen after 3 days of treatment with methylprednisolone, compared with before treatment (82.4\% versus $100 \%$; $\mathrm{p}=0.025$ ) [52] (figure $4 \mathrm{~b}$ ).

\section{Other therapies}

In a small trial $(n=30)$, fewer patients with severe COVID-19 required invasive mechanical ventilation; and the composite end-point of death or invasive mechanical ventilation occurred significantly less frequently in patients receiving auxora than in those receiving standard care (HR 0.23 (95\% CI 0.05-0.96); p<0.05) (figure $4 \mathrm{~b}$ and supplementary table 4) [56]. In another small trial $(\mathrm{n}=20)$, administration of enoxaparin significantly reduced the median number of ventilator-free days compared with low molecular weight/ unfractionated heparin ( 0 versus 15 days; $\mathrm{p}=0.028$ ) and resulted in a higher ratio of successful liberation from mechanical ventilation after respiratory failure (HR 4.0 (95\% CI 1.035-15.053); $\mathrm{p}=0.031$ ) in patients with severe COVID-19 (supplementary table 4) [54]. Trials of ruxolitinib, N-acetylcysteine and rhG-CSF ( $n=43-200)$ showed no significant efficacy in reducing need for ventilation in patients with severe COVID-19 [32, 48, 49] (figure $4 \mathrm{~b}$ and supplementary table 4).

\section{Quality of evidence}

There was variation among trials in terms of the risk of bias in the various domains assessed (supplementary figure 4). Most trials were deemed at low risk of bias in terms of complete reporting of patient and outcomes data. However, most trials were also judged to be at high risk of bias in terms of blinding of participants and researchers to the intervention received. Only four randomised double-blind trials were considered to be at low risk of bias for all domains assessed $[42,44,50,66]$. 

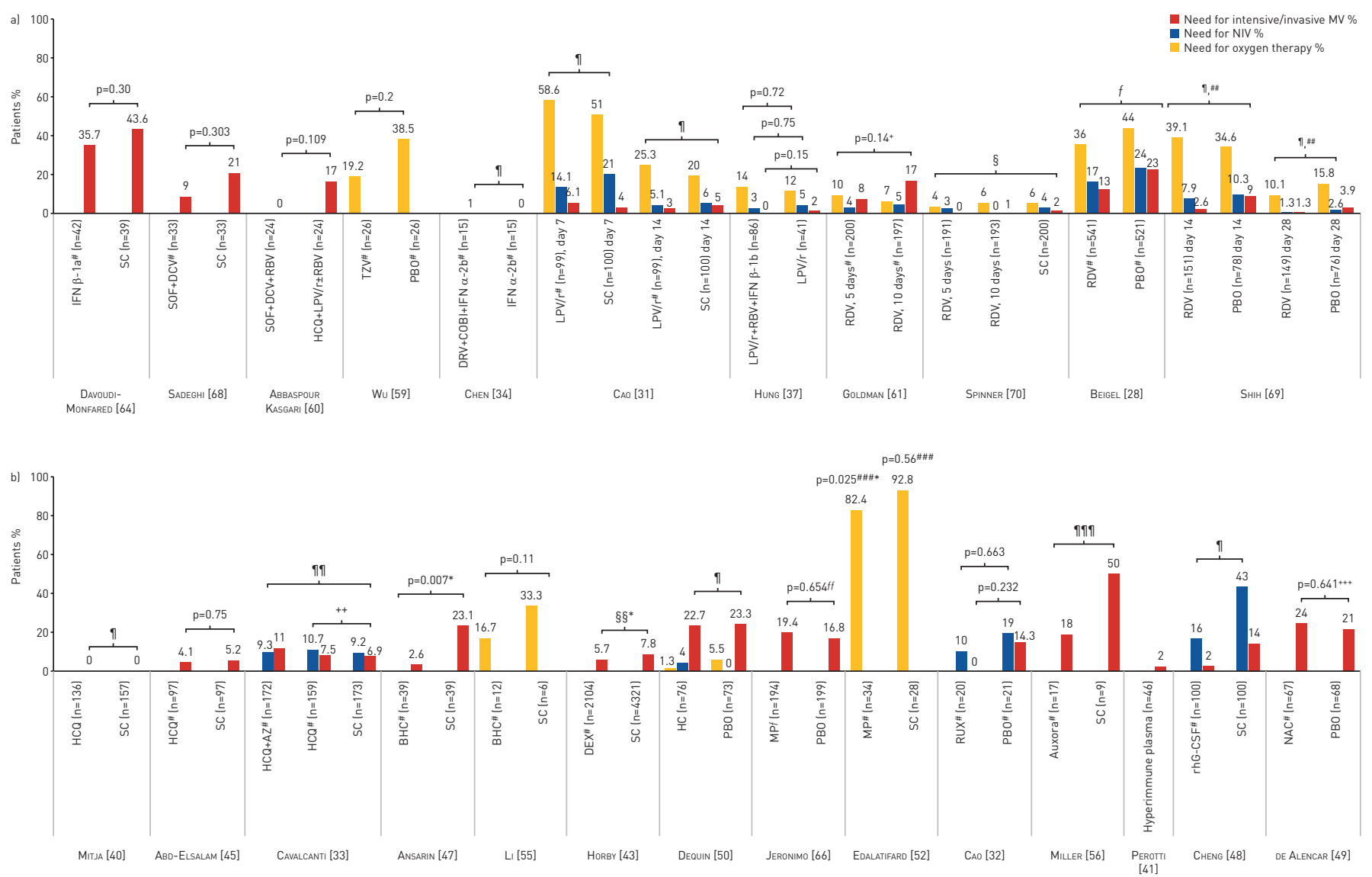

FIGURE 4 Need for ventilation in trials of a) antivirals and b) other therapies included in the qualitative synthesis. Other therapies include antimalarial drugs, mucolytic drugs, anti-inflammatory drugs, anticoagulants, immunomodulatory therapies and repair therapies. Results from two studies are not presented graphically. LI et al. [39] reported need for intensive mechanical ventilation in two (15.4\%) out of 13 severe patients; DefTEREos et al. [36] reported need for ventilation among seven patients who met the primary clinical end-point: in the control group, one (14.3\%) out of seven patients needed noninvasive mechanical ventilation and five (71.4\%) were intubated and ventilated mechanically. The patient in the colchicine group who met the end-point needed invasive mechanical ventilation (MV). NIV: noninvasive ventilation; IFN: interferon; SC: standard care; SOF: sofosbuvir; DCV: daclatasvir; RBV: ribavirin; HCQ: hydroxychloroquine; LPV/r: lopinavir/ritonavir; TZV: triazavirin; PBO: placebo; DRV: darunavir; COBI: cobicistat; RDV: remdesivir; AZ: azithromycin; BHC: bromhexine; DEX: dexamethasone; HC: hydrocortisone; MP: methylprednisolone; RUX: ruxolitinib; rhG-CSF: recombinant human granulocyte colony-stimulating factor; NAC: N-acetylcysteine. ${ }^{\#}$ : treatment administered in addition to SC, as defined by the investigators in each trial; ${ }^{\uparrow}$ : no comparison between groups; ${ }^{+}$: $\mathrm{p}^{-v a l u e}$ for clinical status at day 14 (composite of death and ventilation requirement outcomes); ${ }^{\S}$ : difference in clinical status distribution versus standard care, $0 \mathrm{R} 1.65$ (95\% $\mathrm{Cl}$ 1.09-2.48); ${ }^{f}$ outcomes reported in patients not receiving ventilation at baseline; between-group differences (95\% CI): oxygen -8 (-24--8); NIV/

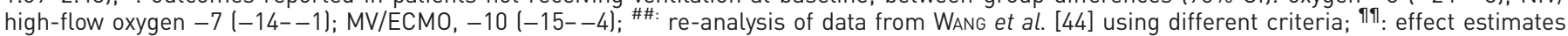
versus SC: need for NIV 1.10 (95\% Cl 0.60-2.03); need for MV 1.77 (95\% Cl 0.81-3.87); ${ }^{++}$: effect estimates versus SC: need for NIV 1.19 (95\% Cl 0.65-2.21); need for MV $1.15(95 \% \mathrm{Cl} 0.49-2.70)$; $\S \S$ : risk ratio: $0.77(95 \% \mathrm{Cl} 0.62-0.95)$; ${ }^{f f}$ : HR $2.6(95 \% \mathrm{Cl}-8.6-13.6)$; \#\#\# : values are in comparison to baseline after 3 days after treatment. After discharge/death, the proportion of patients requiring supplementary oxygen was

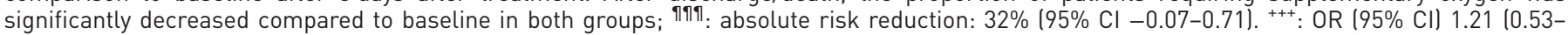
2.72). *: significant $p$-value or other comparison.

\section{Discussion}

This systematic literature review assessed evidence, up to 14 October 2020, for the efficacy of pharmacological interventions for COVID-19 in terms of mortality, hospitalisation and need for ventilation. These outcomes were selected because COVID-19 has caused significant mortality worldwide and continues to impart a substantial burden on healthcare systems [19-22, 71]. Although observational studies have yielded important and useful data, only clinical trials were included in this study to allow a synthesis of the highest quality evidence available for each of the interventions assessed.

Of 42 included trials, all but one assessed a treatment or treatment combination for patients with established COVID-19. One trial assessed the use of hydroxychloroquine as post-exposure prophylaxis in patients ( $\mathrm{n}=821$ ) with high-risk or moderate exposure to COVID-19 versus placebo. There was no difference in mortality or incidence of hospitalisation between groups, but the numbers of patients experiencing these events were very low (no deaths occurred and only one hospitalisation was reported in each group). 
Among the trials assessing treatments in patients with COVID-19, interventions from several treatment classes showed significant effects in improving one or more of mortality, hospitalisation or need for ventilation outcomes. The most consistent effect across all outcomes assessed was reported for the corticosteroid, dexamethasone, in a preliminary report from the Randomised Evaluation of COVid-19 thERapY (RECOVERY) trial (clinicaltrials.gov identifier NCT04381936). The study investigators reported a significant reduction in mortality and a greater probability of discharge alive within 28 days with dexamethasone plus standard care versus standard care alone in patients who were receiving either invasive mechanical ventilation or oxygen at randomisation. Duration of hospitalisation was numerically shorter and the need for invasive mechanical ventilation in patients who were not already receiving this at randomisation was significantly reduced with the addition of dexamethasone to standard care [43].

Other trials of anti-inflammatory drugs included in the present review presented some evidence for efficacy in improving one or more of the outcomes assessed [36, 46, 50, 52, 66]. However, issues reported in these studies included small patient numbers [52,66], potentially being underpowered [50], and late administration of the study treatment in some patients [66]. Since our systematic searches were conducted, further randomised trial evidence has emerged to support the efficacy of colchicine in reducing the composite end-point of death or hospitalisation in outpatients with COVID-19 [72]. Additionally, in an initial report from the international Randomised, Embedded, Multifactorial Adaptive Platform Trial for Community-Acquired Pneumonia (REMAP-CAP) trial (clinicaltrials.gov identifier NCT02735707), the interleukin-6 receptor antagonists tocilizumab and sarilumab improved clinical outcomes, including survival, compared with standard care in critically ill patients with COVID-19 [73]. These findings are promising and, together with further well-designed and adequately powered studies, will increase the body of evidence for anti-inflammatory drugs as treatment for COVID-19.

In this review, findings from trials assessing drugs acting in the early infection or early pulmonary disease phase varied depending on the type of agent evaluated, the disease stage of patients enrolled in the study and the timing of drug administration. Some efficacy in reducing mortality, hospitalisation duration and need for ventilation was seen with IFN therapies [64, 65, 67]; conversely, most trials of lopinavir/ritonavir reported no significant benefit above standard care [31, 39, 62]. Similarly, and in line with conclusions from published meta-analyses $[74,75]$ and a large randomised trial published after the cut-off dates of our systematic searches [76], trials that assessed hydroxychloroquine, an antimalarial drug, or its derivatives reported no impact of these interventions on any of the outcomes of interest. Moreover, high doses of chloroquine diphosphate were associated with increased lethality compared with low doses in one trial [29].

Trials that assessed remdesivir compared with placebo or standard care also failed to show a clear benefit in terms of reducing mortality, although one trial showed a reduction in median hospitalisation duration and need for ventilation with remdesivir compared with placebo [28]. Despite the lack of strong evidence of efficacy with remdesivir, it is notable that this drug has received endorsement from regulatory bodies for treating COVID-19 on the basis of data showing improved time to recovery and fewer adverse events in remdesivir-treated patients versus placebo in two clinical trials included in the present review, particularly in patients with less severe disease [28, 44]. Consistent with these findings, a re-analysis of data from one trial [44] suggested that remdesivir induced good responses in patients with moderately severe, rather than critical, disease at enrolment [69]. Taken together, the data suggest that remdesivir may improve recovery time if administered early enough in the disease course; appropriate selection of patients with early-stage disease in future trials of remdesivir may help to confirm this hypothesis. Additionally, the combination of remdesivir with other agents may be more efficacious than remdesivir alone, as demonstrated in a randomised clinical trial of remdesivir plus baricitinib, published after the cut-off date for the systematic searches in this review [77].

Collectively, the findings from trials of drugs acting early in the pathophysiological course of COVID-19 summarised in the present review are broadly in line with those from the SOLIDARITY trial. This international, randomised trial of COVID-19 treatments concluded that all four treatments evaluated (remdesivir, hydroxychloroquine, lopinavir/ritonavir and IFNs) had little or no effect on overall mortality, initiation of ventilation and hospitalisation duration in patients hospitalised with COVID-19 [78]. A notable difference is that some studies in the present review did show some efficacy of IFN therapies in reducing mortality and improving hospitalisation outcomes [64, 65, 67], suggesting that these therapies may warrant further investigation. Indeed, findings from a phase 2 pilot study published after the cut-off date for the systematic searches in this review reported that SNG001, an inhaled INF- $\beta-1$ a formulation, was associated with greater odds of improvement and more rapid recovery versus placebo in patients hospitalised with COVID-19 [79]. A global phase 3 trial investigating SNG001 was initiated in January 2021 (EudraCT number: 2020-004743-83).

A key finding from this review was the relationship between the disease phase of patients with COVID-19 and the efficacy of interventions. Several studies that investigated agents targeting processes early in the 
disease course of COVID-19, such as viral replication, showed improved efficacy in patients who received early treatment compared with late treatment $[31,37,44,67]$. Similarly, in a report from the RECOVERY trial, the mortality benefit of dexamethasone was greatest in patients with either the most severe disease or with the longest duration of symptoms [43], which is consistent with it acting during the inflammatory phase of the disease. These findings highlight the need for COVID-19 therapies to be tailored to patients with disease stage and severity appropriate to the mechanism of action of the intervention, as has been reported previously [80], in order for maximum efficacy to be attained.

A small number of trials included in the present review reported on other types of therapies, including kinase or calcium release-activated calcium channel inhibitor, anticoagulants, convalescent plasma and other immunomodulatory or repair therapies [32, 38, 41, 48, 49,54,56]. Some studies showed either a significant efficacy effect or a trend towards improved efficacy [32, 38, 48, 49, 54, 56]; however, in general the studies were of low quality and further clinical trials are required for a more conclusive demonstration of efficacy. In particular, although convalescent plasma has received emergency use authorisation from the US FDA, the decision was controversial, owing to a lack of robust supporting data [13]. Only one study of convalescent plasma $(n=103)$ was included in the current review, and did not show significant improvements in any of the outcomes assessed compared with standard care [38]. Notably, a study published after the systematic searches for this systematic literature review were conducted reported a benefit of high-titre convalescent plasma in reducing progression to severe illness when administered to adults aged $\geqslant 75$ years within $72 \mathrm{~h}$ of mild symptoms [81]. These findings support the premise that the timing of treatment and patient selection may be important determinants of efficacy with COVID-19 therapies. Ongoing randomised studies, including the UK's RECOVERY trial, will be important in providing further evidence to clarify whether convalescent plasma offers any benefit in treating COVID-19.

It was also notable that only one small clinical trial $(n=20)$ [54] reported on an anticoagulant, enoxaparin, despite many institutions having adopted anticoagulant therapy as standard care for hospitalised patients with COVID-19. The data presented were promising and showed a reduced need for ventilation compared with patients receiving prophylactic anticoagulation. These findings support those from a recently published large observational study, which reported an association between anticoagulant use and lower in-hospital mortality and intubation rates in patients hospitalised with COVID-19 [82]. Upcoming randomised trials will provide valuable data regarding the optimal type, duration and dose of anticoagulants for different patients.

Strengths of this systematic literature review include the comprehensive search strategy, rigorous screening methodology and quality assessment of included studies. Although several other reviews of COVID-19 therapies have been published [7, 83, 84], we believe that our overview of the current state of the field makes an important contribution to the existing body of evidence, particularly by considering the efficacy of current treatments in the context of the disease phase in which they are used.

Limitations include the exclusion of non-English articles from searches, which may have resulted in some relevant trials not being identified. Additionally, the searches were conducted in three major databases but did not include smaller or country-specific databases. Congress abstracts were excluded on the basis of their data being preliminary and not peer reviewed. Owing to time constraints and the fast-moving nature of the field, a review of citations in the reference lists of published systematic literature reviews and meta-analysis was not conducted; and, similarly, the review will not have captured relevant studies published after the date of the final search update.

Our ability to analyse and compare the data collected was limited by the significant variation between the trials identified, owing to differences in study design, blinding of participants, severity of illness of participants, drug doses and timing, comparators, follow-up times and outcome definitions. Most trials administered interventions in addition to standard care, which varies between countries and has changed during the pandemic as the body of evidence for the efficacy and safety of different therapies has accumulated. Similarly, the overall lack of high-quality evidence for all interventions makes it difficult to make comparisons between interventions.

Most of the trials included in this systematic literature review were assessed as having a high risk of bias in one or more of the domains evaluated, meaning that the results of the analysis of all outcomes should be interpreted with caution. A variety of factors contributed to this: some trials were pilot $[34,55,57,59]$ or proof-of-concept [41] trials, including small patient numbers and might not have been optimally designed to show an improvement in the study outcomes. Similarly, some trials were under-powered to show significant differences between interventions [32, 38, 40, 44, 54, 62, 63]. Some trials did not include a randomised placebo control group and many trials were not double-blinded. Moreover, the follow-up period in most trials was between 14 and 28 days, and most studies did not report any longer term data. 
This might have limited the ability to assess mortality and hospital discharge, particularly in patients with severe COVID-19 who often have a prolonged duration of illness.

These findings are in agreement with those reported in a living systematic review and meta-analysis of drug treatments for COVID-19, last updated in September 2020 [84]. The authors concluded that the effectiveness of most of the interventions that they assessed was uncertain, owing to the small numbers of patients enrolled in randomised controlled trials at the time and important limitations of the study designs [84]. A European Medicines Agency statement noted that small studies and compassionate programmes are unlikely to generate the required level of evidence to define the best treatment options for COVID-19 and stressed the need for multi-arm randomised controlled trials of interventions [85]. However, it should be acknowledged that the COVID-19 global public health emergency has presented an urgent need for clinical data on available interventions for the disease, with the US FDA granting some substances emergency use authorisation (table 1). Thus, the available data are valuable for the purpose of practical clinical decision making.

Although no relevant data from vaccine, antibody and other novel COVID-19-specific interventions were available at the time of conducting this review, interim data from several phase 3 clinical trials of vaccines have shown high efficacy in preventing symptomatic COVID-19 infection and protection against severe disease [86-88]. Further findings from ongoing clinical trials are awaited with interest.

\section{Conclusions}

This systematic literature review summarises evidence regarding efficacy of pharmacological interventions in terms of mortality, hospitalisation and need for ventilation in patients with COVID-19, and highlights the need for adequately powered, well-designed clinical trials to increase the quality of available evidence. The summary of findings also suggests the need to use interventions appropriate for the disease stage of COVID-19, to maximise treatment efficacy.

Acknowledgements: Editorial support was provided by Rachael Cazaly (Core Medica, London, UK), supported by AstraZeneca according to Good Publication Practice guidelines. The sponsor was involved in the study design, analysis and interpretation of data. However, ultimate responsibility for opinions, conclusions and data interpretation lies with the authors.

Conflict of interest: T. Welte reports personal fees, fees for lectures and consultancy fees from AstraZeneca, Basilea, Biotest, Bayer, Boehringer, GlaxoSmithKline, Janssens, Merck Sharp \& Dohme, Novartis, Pfizer, Roche and Sanofi Aventis, outside the submitted work. L.J. Ambrose reports other funding from AstraZeneca, during the conduct of the study. G.C. Sibbring reports other funding from AstraZeneca, during the conduct of the study. S. Sheikh was an employee and shareholder of AstraZeneca at the time of manuscript preparation. H. Müllerová is an employee and shareholder of AstraZeneca. I. Sabir is an employee and shareholder of AstraZeneca.

\section{References}

1 Wiersinga WJ, Rhodes A, Cheng AC, et al. Pathophysiology, transmission, diagnosis, and treatment of coronavirus disease 2019 (COVID-19): a review. JAMA 2020; 324: 782-793.

2 Institute for Health Metrics and Evaluation. COVID-19 projections. https://covid19.healthdata.org/global? view=total-deaths\&tab=trend Date last accessed: 28 January 2021.

3 Gavriatopoulou M, Korompoki E, Fotiou D, et al. Organ-specific manifestations of COVID-19 infection. Clin Exp Med 2020; 20: 493-506.

4 Siddiqi HK, Mehra, MR. COVID-19 illness in native and immunosuppressed states: a clinical-therapeutic staging proposal. J Heart Lung Transplant 2020; 39: 405-407.

5 Hoffmann M, Kleine-Weber H, Schroeder S, et al. SARS-CoV-2 cell entry depends on ACE2 and TMPRSS2 and is blocked by a clinically proven protease inhibitor. Cell 2020; 181: 271-280.e278.

6 Inciardi RM, Solomon SD, Ridker PM, et al. Coronavirus 2019 disease (COVID-19), systemic inflammation, and cardiovascular disease. J Am Heart Assoc 2020; 9: e017756.

7 Gudadappanavar AM, Benni J. An evidence-based systematic review on emerging therapeutic and preventive strategies to treat novel coronavirus (SARS-CoV-2) during an outbreak scenario. J Basic Clin Physiol Pharmacol 2020; 31 .

8 National Institutes of Health. COVID-19 Treatment Guidelines Panel. Coronavirus disease 2019 (COVID-19) treatment guidelines. www.covid19treatmentguidelines.nih.gov/ Date last accessed: 28 January 2021.

9 British Medical Journal. BMJ Best Practice. Coronavirus disease 2019 (COVID-19): emerging treatments. https:// bestpractice.bmj.com/topics/en-gb/3000201/emergingtxs Date last accessed: 28 January 2021.

10 European Medicines Agency. Treatments and vaccines for COVID-19. www.ema.europa.eu/en/human-regulatory/ overview/public-health-threats/coronavirus-disease-covid-19/treatments-vaccines/ treatments-vaccines-covid-19-authorised-medicines Date last accessed: 22 September 2020.

11 Food and Drug Administration. Coronavirus Disease 2019 (COVID-19) EUA Information. www.fda.gov/ emergency-preparedness-and-response/mcm-legal-regulatory-and-policy-framework/ emergency-use-authorization\#coviddrugs Date last accessed: 28 January 2021.

12 Food and Drug Administration. FDA approves first treatment for COVID-19. www.fda.gov/news-events/ press-announcements/fda-approves-first-treatment-covid-19 Date last updated: 22 October 2020; date last accessed: 28 January 2021.

13 Estcourt L, Roberts D. Convalescent plasma for covid-19. BMJ 2020; 370: m3516. 
14 Wu SL, Mertens AN, Crider YS, et al. Substantial underestimation of SARS-CoV-2 infection in the United States. Nat Commun 2020; 11: 4507.

15 Verity R, Okell LC, Dorigatti I, et al. Estimates of the severity of coronavirus disease 2019: a model-based analysis. Lancet Infect Dis 2020; 20: 669-677.

16 Li J, Huang DQ, Zou B, et al. Epidemiology of COVID-19: a systematic review and meta-analysis of clinical characteristics, risk factors, and outcomes. J Med Virol 2021; 93: 1449-1458.

17 Huang C, Wang Y, Li X, et al. Clinical features of patients infected with 2019 novel coronavirus in Wuhan, China. Lancet 2020; 395: 497-506.

18 Docherty A, Harrison E, Green C, et al. Features of 20133 UK patients in hospital with covid-19 using the ISARIC WHO Clinical Characterisation Protocol: prospective observational cohort study. BMJ 2020; 369: m1985.

19 European Data Portal. Pressure on healthcare systems: coping with demand for ICU and hospital beds. www. europeandataportal.eu/en/impact-studies/covid-19/

pressure-healthcare-systems-coping-demand-icu-and-hospital-beds Date last accessed: 22 September 2020.

20 Miller IF, Becker AD, Grenfell BT, et al. Disease and healthcare burden of COVID-19 in the United States. Nat Med 2020; 26: 1212-1217.

21 Emanuel EJ, Persad G, Upshur R, et al. Fair allocation of scarce medical resources in the time of Covid-19. $N$ Engl J Med 2020; 382: 2049-2055.

22 White DB, Lo B. A framework for rationing ventilators and critical care beds during the COVID-19 pandemic. JAMA 2020; 323: 1773-1774.

23 Karagiannidis C, Mostert C, Hentschker C, et al. Case characteristics, resource use, and outcomes of 10021 patients with COVID-19 admitted to 920 German hospitals: an observational study. Lancet Respir Med 2020; 8: 853-862.

24 Higgins JP. Cochrane handbook for systematic reviews of interventions version 6.1 (updated September 2020). www.training.cochrane.org/handbook Date last accessed: 28 January 2021.

25 Centre for Reviews and Dissemination. CRD's guidance for undertaking reviews in healthcare. www.york.ac.uk/ crd/SysRev/!SSL!/WebHelp/SysRev3.htm Date last updated: January 2009; date last accessed: 28 January 2021.

26 Norwegian Institute of Public Health. NIPH systematic and living map on COVID-19 evidence. www.nornesk.no/ forskningskart/NIPH_mainMap.html Date last accessed: 28 January 2021.

27 Higgins JP, Altman DG, Gotzsche PC, et al. The Cochrane collaboration's tool for assessing risk of bias in randomised trials. BMJ 2011; 343: d5928.

28 Beigel JH, Tomashek KM, Dodd LE, et al. Remdesivir for the treatment of Covid-19 - final report. N Engl J Med 2020; 383: 1813-1826.

29 Borba MGS, Val FFA, Sampaio VS, et al. Effect of high vs low doses of chloroquine diphosphate as adjunctive therapy for patients hospitalized with severe acute respiratory syndrome coronavirus 2 (SARS-CoV-2) infection. JAMA Netw Open 2020; 3: e208857.

30 Boulware DR, Pullen MF, Bangdiwala AS, et al. A randomized trial of hydroxychloroquine as postexposure prophylaxis for Covid-19. N Engl J Med 2020; 383: 517-525.

31 Cao B, Wang Y, Wen D, et al. A trial of lopinavir-ritonavir in adults hospitalized with severe Covid-19. $N$ Engl J Med 2020; 382: 1787-1799.

32 Cao Y, Wei J, Zou L, et al. Ruxolitinib in treatment of severe coronavirus disease 2019 (COVID-19): a multicenter, single-blind, randomized controlled trial. J Allergy Clin Immunol 2020; 146: 137-146.e133.

33 Cavalcanti A, Zampieri F, Rosa R, et al. Hydroxychloroquine with or without azithromycin in mild-to-moderate Covid-19. N Engl J Med 2020; 383: 2041-2052.

34 Chen J, Xia L, Liu L, et al. Antiviral activity and safety of darunavir/cobicistat for the treatment of COVID-19. Open Forum Infect Dis 2020; 7: ofaa241.

35 Davoodi L, Abedi SM, Salehifar E, et al. Febuxostat therapy in outpatients with suspected COVID-19: a clinical trial. Int J Clin Pract 2020; 74: e13600.

36 Deftereos S, Giannopoulos G, Vrachatis D, et al. Effect of colchicine vs standard care on cardiac and inflammatory biomarkers and clinical outcomes in patients hospitalized with coronavirus disease 2019: the GRECCO-19 randomized clinical trial. JAMA Netw Open 2020; 3: e2013136.

37 Hung IF-N, Lung K-C, Tso EY-K, et al. Triple combination of interferon beta-1b, lopinavir-ritonavir, and ribavirin in the treatment of patients admitted to hospital with COVID-19: an open-label, randomised, phase 2 trial. Lancet 2020; 395: 1695-1704.

$38 \mathrm{Li} \mathrm{L}$, Zhang W, Hu Y, et al. Effect of convalescent plasma therapy on time to clinical improvement in patients with severe and life-threatening COVID-19. JAMA 2020; 324: 460-470.

39 Li Y, Xie Z, Lin W, et al. Efficacy and safety of lopinavir/ritonavir or arbidol in adult patients with mild/moderate COVID-19: an exploratory randomized controlled trial. Med 2020; 1: 105-113.

40 Mitja O, Corbacho-Monne M, Ubals M, et al. Hydroxychloroquine for early treatment of adults with mild Covid-19: a randomized-controlled trial. Clin Infect Dis 2020; https://doi.org/10.1093/cid/ciaa1009.

41 Perotti C, Baldanti F, Bruno R, et al. Mortality reduction in 46 severe Covid-19 patients treated with hyperimmune plasma. A proof of concept single arm multicenter trial. Haematologica 2020; 105: 2834-2840.

42 Skipper CP, Pastick KA, Engen NW, et al. Hydroxychloroquine in nonhospitalized adults with early COVID-19: a randomized trial. Ann Intern Med 2020; 173: 623-631.

43 The RECOVERY Collaborative Group. Dexamethasone in hospitalized patients with Covid-19 - preliminary report. N Engl J Med 2021; 384: 693-704.

44 Wang Y, Zhang D, Du G, et al. Remdesivir in adults with severe COVID-19: a randomised, double-blind, placebo-controlled, multicentre trial. Lancet 2020; 395: 1569-1578.

45 Abd-Elsalam S, Esmail E, Khalaf M, et al. Hydroxychloroquine in the treatment of COVID-19: a multicenter randomized controlled study. Am J Trop Med Hyg 2020; 103: 1635-1639.

46 Angus D, Derde L, Al-Beidh F, et al. Effect of hydrocortisone on mortality and organ support in patients with severe COVID-19: the REMAP-CAP COVID-19 corticosteroid domain randomized clinical trial. JAMA 2020; 324 $1317-1329$.

47 Ansarin K, Tolouian R, Ardalan M, et al. Effect of bromhexine on clinical outcomes and mortality in COVID-19 patients: a randomized clinical trial. Bioimpacts 2020; 10: 209-215. 
48 Cheng L, Guan W, Duan C, et al. Effect of recombinant human granulocyte colony-stimulating factor for patients with coronavirus disease 2019 (COVID-19) and lymphopenia: a randomized clinical trial. JAMA Intern Med 2020; 181: 71-78.

49 de Alencar J, Moreira C, Müller A, et al. Double-blind, randomized, placebo-controlled trial with N-acetylcysteine for treatment of severe acute respiratory syndrome caused by COVID-19. Clin Infect Dis 2020; https://doi.org/10. 1093/cid/ciaal443.

50 Dequin P, Heming N, Meziani F, et al. Effect of hydrocortisone on 21-day mortality or respiratory support among critically ill patients with COVID-19: a randomized clinical trial. JAMA 2020; 324: 1298-1306.

51 Doi Y, Hibino M, Hase R, et al. A prospective, randomized, open-label trial of early versus late favipiravir in hospitalized patients with COVID-19. Antimicrob Agents Chemother 2020; 64: e01897-20.

52 Edalatifard M, Akhtari M, Salehi M, et al. Intravenous methylprednisolone pulse as a treatment for hospitalised severe COVID-19 patients: results from a randomised controlled clinical trial. Eur Respir J 2020; 56: 2002808.

53 Furtado R, Berwanger O, Fonseca H, et al. Azithromycin in addition to standard of care versus standard of care alone in the treatment of patients admitted to the hospital with severe COVID-19 in Brazil (COALITION II): a randomised clinical trial. Lancet 2020; 396: 959-967.

54 Lemos ACB, do Espírito Santo DA, Salvetti MC, et al. Therapeutic versus prophylactic anticoagulation for severe COVID-19: a randomized phase II clinical trial (HESACOVID). Thromb Res 2020; 196: 359-366.

55 Li T, Sun L, Zhang W, et al. Bromhexine hydrochloride tablets for the treatment of moderate COVID-19: an open-label randomized controlled pilot study. Clin Transl Sci 2020; 13: 1096-1102.

56 Miller J, Bruen C, Schnaus M, et al. Auxora versus standard of care for the treatment of severe or critical COVID-19 pneumonia: results from a randomized controlled trial. Critical Care 2020; 24: 502.

57 Ren Z, Luo H, Yu Z, et al. A randomized, open-label, controlled clinical trial of azvudine tablets in the treatment of mild and common COVID-19, a pilot study. Adv Sci (Weinh) 2020; 7: 2001435.

58 Wang M, Zhao Y, Hu W, et al. Treatment of COVID-19 patients with prolonged post-symptomatic viral shedding with leflunomide - a single-center, randomized, controlled clinical trial. Clin Infect Dis 2020; https://doi.org/10. $1093 /$ cid/ciaal 1417.

59 Wu X, Yu K, Wang Y, et al. Efficacy and safety of triazavirin therapy for coronavirus disease 2019: a pilot randomized controlled trial. Engineering (Beijing) 2020; 6: 1185-1191.

60 Abbaspour Kasgari H, Moradi S, Shabani A, et al. Evaluation of the efficacy of sofosbuvir plus daclatasvir in combination with ribavirin for hospitalized COVID-19 patients with moderate disease compared with standard care: a single-centre, randomized controlled trial. J Antimicrob Chemother 2020; 75: 3373-3378.

61 Goldman JD, Lye DCB, Hui DS, et al. Remdesivir for 5 or 10 days in patients with severe Covid-19. N Engl J Med 2020; 383: 1827-1837.

62 Huang Y-Q, Tang S-Q, Xu X-L, et al. No statistically apparent difference in antiviral effectiveness observed among ribavirin plus interferon-alpha, lopinavir/ritonavir plus interferon-alpha, and ribavirin plus lopinavir/ritonavir plus interferon-alpha in patients with mild to moderate coronavirus disease 2019: results of a randomized, open-labeled prospective study. Front Pharmacol 2020; 11: 1071.

63 Tang W, Cao Z, Han M, et al. Hydroxychloroquine in patients with mainly mild to moderate coronavirus disease 2019: open label, randomised controlled trial. BMJ 2020; 369: m1849.

64 Davoudi-Monfared E, Rahmani H, Khalili $\mathrm{H}$, et al. A randomized clinical trial of the efficacy and safety of interferon $\beta$-1a in treatment of severe COVID-19. Antimicrob Agents Chemother 2020; 64: e01061-20.

65 Fu W, Liu Y, Liu L, et al. An open-label, randomized trial of the combination of IFN- $\kappa$ plus TFF2 with standard care in the treatment of patients with moderate COVID-19. EClinicalMedicine 2020; 27: 100547.

66 Jeronimo C, Farias M, Val F, et al. Methylprednisolone as adjunctive therapy for patients hospitalized with COVID-19 (metcovid): a randomised, double-blind, phase IIb, placebo-controlled trial. Clin Infect Dis 2020; https://doi.org/10.1093/cid/ciaa1177.

67 Rahmani H, Davoudi-Monfared E, Nourian A, et al. Interferon $\beta-1 \mathrm{~b}$ in treatment of severe COVID-19: a randomized clinical trial. Int Immunopharmacol 2020; 88: 106903.

68 Sadeghi A, Ali Asgari A, Norouzi A, et al. Sofosbuvir and daclatasvir compared with standard of care in the treatment of patients admitted to hospital with moderate or severe coronavirus infection (COVID-19): a randomized controlled trial. J Antimicrob Chemother 2020; 75: 3379-3385.

69 Shih WJ, Shen X, Zhang P, et al. Remdesivir is effective for moderately severe patients: a re-analysis of the first double-blind, placebo-controlled, randomized trial on remdesivir for treatment of severe COVID-19 patients conducted in Wuhan city. Open Access J Clin Trials 2020; 12: 15-21.

70 Spinner CD, Gottlieb RL, Criner GJ, et al. Effect of remdesivir vs standard care on clinical status at 11 days in patients with moderate COVID-19: a randomized clinical trial. JAMA 2020; 324: 1048-1057.

71 World Health Organization. WHO coronavirus disease (COVID-19) dashboard. https://covid19.who.int/ Date last accessed: 22 September 2020.

72 Tardif J-C, Bouabdallaoui N, L'Allier PL, et al. Efficacy of colchicine in non-hospitalized patients with COVID-19. medRxiv 2021; preprint [https://doi.org/10.1101/2021.01.26.21250494].

73 Gordon AC, Mouncey PR, Al-Beidh F, et al. Interleukin-6 receptor antagonists in critically ill patients with Covid-19 - preliminary report. medRxiv 2021; preprint [https://doi.org/10.1101/2021.01.07.21249390].

74 Pathak DSK, Salunke DAA, Thivari DP, et al. No benefit of hydroxychloroquine in COVID-19: results of systematic review and meta-analysis of randomized controlled trials. Diabetes Metab Syndr 2020; 14: 1673-1680.

75 Siordia JA, Jr, Bernaba M, Yoshino K, et al. Systematic and statistical review of coronavirus disease 19 treatment trials. SN Compr Clin Med 2020; 2: 1120-1131.

76 Self WH, Semler MW, Leither LM, et al. Effect of hydroxychloroquine on clinical status at 14 days in hospitalized patients with COVID-19: a randomized clinical trial. JAMA 2020; 324: 2165-2176.

77 Kalil AC, Patterson TF, Mehta AK, et al. Baricitinib plus remdesivir for hospitalized adults with Covid-19. $N$ Engl J Med 2021; 384: 795-807.

78 WHO Solidarity Trial Consortium, Pan H, Peto R, et al. Repurposed antiviral drugs for Covid-19 - interim WHO solidarity trial results. N Engl J Med 2021; 384: 497-511. 
79 Monk PD, Marsden RJ, Tear VJ, et al. Safety and efficacy of inhaled nebulised interferon beta-1a (SNG001) for treatment of SARS-CoV-2 infection: a randomised, double-blind, placebo-controlled, phase 2 trial. Lancet Respir Med 2021; 9: 196-206.

80 Razmi M, Hashemi F, Gheytanchi E, et al. Immunomodulatory-based therapy as a potential promising treatment strategy against severe COVID-19 patients: a systematic review. Int Immunopharmacol 2020; 88: 106942.

81 Libster R, Perez Marc G, Wappner D, et al. Early high-titer plasma therapy to prevent severe Covid-19 in older adults. N Engl J Med 2021; 384: 610-618.

82 Nadkarni GN, Lala A, Bagiella E, et al. Anticoagulation, mortality, bleeding and pathology among patients hospitalized with COVID-19: a single health system study. J Am Coll Cardiol 2020; 76: 1815-1826.

83 Juul S, Nielsen EE, Feinberg J, et al. Interventions for treatment of COVID-19: a living systematic review with meta-analyses and trial sequential analyses (The LIVING Project). PLoS Med 2020; 17: e1003293.

84 Siemieniuk RA, Bartoszko JJ, Ge L, et al. Drug treatments for covid-19: living systematic review and network meta-analysis. BMJ 2020; 370: m2980.

85 European Medicines Agency. A call to pool EU research resources into large-scale, multi-centre, multi-arm clinical trials against COVID-19. www.ema.europa.eu/en/documents/other/call-pool-eu-research-resources-large-scalemulti-centre-multi-arm-clinical-trials-against-covid-19_en.pdf Date last updated: 16 March 2020; date last accessed: 28 January 2021.

86 Baden LR, El Sahly HM, Essink B, et al. Efficacy and safety of the mRNA-1273 SARS-CoV-2 vaccine. N Engl J Med 2021; 384: 403-416.

87 Polack FP, Thomas SJ, Kitchin N, et al. Safety and efficacy of the BNT162b2 mRNA Covid-19 vaccine. $N$ Engl J Med 2020; 383: 2603-2615.

88 Voysey M, Clemens SAC, Madhi SA, et al. Safety and efficacy of the ChAdOx1 nCoV-19 vaccine (AZD1222) against SARS-CoV-2: an interim analysis of four randomised controlled trials in Brazil, South Africa, and the UK. Lancet 2021; 397: 99-111. 